Economic Theory manuscript No.

(will be inserted by the editor)

\title{
Strategies in the Principal-Agent Model
}

\author{
James Mirrlees · Roberto C. Raimondo
}

Received: date / Accepted: date

\begin{abstract}
In this paper we study the principal-agent problem. It is well-known that in continuous-time it is possible to prove the existence of an equilibrium $\left(u^{*}, S^{*}\right)$ in a considerably general setting, however little is known about the strategies themselves. Our goal is to present a universal way to construct these strategies as limiting strategies of a very simple type.
\end{abstract}

Keywords Agency Theory, Principal-Agent Problems, Incentive Schemes, Brownian Motion.

JEL Classification: D81

We are very grateful to Robert Anderson, Shachar Kariv, Donald MacLaren, John Quah, Bruno Strulovici, Dimitrios Tsomocos and John Zhou for very helpful discussions and comments. Preliminary versions of this paper were presented at Said Business School in November 2008, University of California at Berkeley in November 2009, and University of California at Los Angeles in November 2009 and at the SAET Conference in Kos 2007; we are grateful to all participants for their comments.

The work of both authors was supported by a Visiting Research Scholar Grant from the University of Melbourne.

Correspondence to: Roberto Raimondo.

James A. Mirrlees

Chinese University of Hong Kong

Hong Kong

E-mail: jam28@cam.ac.uk

Roberto C. Raimondo

Department of Economics

University of Melbourne

Melbourne, AUSTRALIA

and

Department of Mathematics and Applications

Universita degli Studi Milano-Bicocca

Milano, ITALY

E-mail: rraim@unimelb.edu.au 


\section{Introduction}

A typical principal-agent situation under moral hazard is usually modelled in the following way: the agent must manage some form of assets while receiving a salary to do so. The principal will compensate the agent at the end and cannot monitor the effort, leading the agent to maximize her/his own wealth. Therefore, in a world of moral hazard, the participation constraint is not the only condition to be taken into account.

If the agent has to perform her/his task in a continuous-time setting, then we usually allow the agent to control the drift of a diffusion process and, under certain assumptions, it is possible to show that there exists a salary and a control such that they are the equilibrium of the game.

Many papers have been written along this line. They are all very interesting and they all originated from the seminal paper of Holmström and Milgrom. In this paper, where the agent controls a Brownian motion with a linear trend (see [14]), they find a natural resolution of Mirrlees' nonexistence result (1974) and a very sharp conclusion: at equilibrium the principal offers a salary which is a linear function of profit and elicits a constant effort from the agent. It would be desirable to have a way to get information about the strategy played at equilibrium when the output is a more general process, since we know that in many situations, Brownian motion with a drift is not a modelling option; for example, when the process must be positive we cannot use Brownian motion with drift.

So far, little attention has been paid to the mathematical underpinning of this problem, but there are two important exceptions, the paper of Sung (see [33]) and the paper of Hellwig and Schmidt (see [12]). This direction of investigation is very interesting since it has the potential to help us to answer one of the main questions about this kind of game-theoretic model, namely which kind of strategies the players use at the equilibrium $\left(u^{*}, S^{*}\right)$, where $S^{*}$ is the salary paid by the principal to the agent who exerts $u^{*}$ effort. In fact, as we are not in a full information framework the strategies are not trivial. Very little is known about this aspect of the problem and only one case has been computed so far: the linear case (see [14]).

In Schaettler and Sung's paper there is a rigorous characterization, via a fully justified first-order approach, of the pair of strategies $\left(u^{*}, S^{*}\right)$ which characterizes the equilibrium of the game. This is our starting point. The characterization for the salary $S^{*}$ is given, among others things, in terms of a stochastic integral, and the stochastic integral is also a function of the optimal control. However, the characterization of the optimal control, crucial to construct even the salary, is given in terms of a nonlinear partial differential equation (PDE thereafter). This is not surprising but certainly useful. In fact, it is well-known that many types of optimal control problems generate some kind of PDE and they are very important from the heuristic point-of-view. However, usually they are not linear PDEs; therefore, very little is known about solutions and ways to get them. Hence they are, in a certain sense, like black boxes. 
It must be underlined that there are two significant factors that make this model rather complex to approach, namely, the presence of a final or expiration date for the contract and the fact that we do not put any restriction on the number of sources of information and on the efforts components. In turn, we will discuss both aspects. The presence of the final date is, in our opinion, very important. In fact, this is a common characteristic for many contracts and to deny this fact is simply unacceptable. Of course to remove this modelling condition makes the analysis simpler since it is possible to use the short cut of statistical stationarity when we remove a boundary or final date condition, but, in our opinion, this model of contract (without a fixed terminal date) is not the only one of interest in economics. Moreover, it is quite easy to think of an interesting situation where the final date or expiration is important in influencing the output and the effort. For a simple example, just think of the pricing of financial contracts in relation to the expiration date.

Now we turn to the second complication. We think that several sources of information are a very interesting aspect of the asymmetric situation and we prefer to approach the problem in its generality rather than working with ad hoc situations. Again, if we restrict ourselves to one source, or a few fluke cases with two sources of information, we may reduce everything to an ordinary differential equation. Of course, it would make things more tractable if we did not include one of these modelling aspects, several sources of uncertainty and no final date (see [32]). However, we are interested in studying contracts with a final date and many sources of uncertainty. Actually, the fact that we do not need to put any of these restrictions on our model suggests that the techniques proposed in this paper are quite valuable and could be applied in many different contexts.

Our main goal therefore is to present a method to construct the optimal control $u^{*}$ and the optimal salary in a reasonably general case, which means when the output satisfies a reasonably arbitrary stochastic differential equation (SDE). To achieve our goal we do not propose to apply the techniques of PDE to the Principal-Agent game. Instead, we prefer to apply a probabilistic method based on the PDE itself. Our approach is to replace the diffusion process with a much simpler process, which is constructed by interpolating in continuous-time and in a nice fashion a discrete-time process, which is not, in general, a simple random walk. Of course we expect the substitution to lead to a much simpler optimal control problem, namely a discrete one, and finally, by iterating this substitution, we would like to see the sequence of solutions converging to the value we are looking for. Actually, this discretization is the critical issue as it is possible to show that a wrong discretization procedure would not guarantee convergence. Therefore, we will solve the discrete problem and show that there is convergence in the appropriate space. In this respect our paper is also related to the paper of Hellwig and Schmidt; the main goal of their paper was to study the relationship between the continuous and the discrete version of the principal-agent problem. Our discretization procedure is quite different from the one used by Hellwig and Schmidt or by Biais, Mariotti, G. Plantin and Rochet in [3], though. 
The solution of the problem of which discretization to use is in the notion of local consistency for the discrete process. Roughly speaking, this condition forces the discrete process to look like the continuous one, at least locally. The trick to constructing the locally consistent process turns out to be extremely intuitive; we re-write a certain PDE induced by the process and the control problem in a discrete fashion and from there it is quite natural to construct the transition probabilities. Our approach is in three steps.

In the first step we construct a finite state space process in order to discretize the process, that is the solution of the SDE. As we outlined earlier, we do this in order to transfer the problem from continuous to discrete time. In the second step we discretize the optimal control problem. We solve the optimization problem for each level of discretization, which is rather simple, and we construct a procedure to find the discrete optimal control $u_{n}$. Then we set the stage to show convergence, by studying the sequence of optimal controls and the sequence of discretized stochastic processes. We also show that the sequence of controls $u_{n}$ converges to the solution $u^{*}$ of the original optimal control and that the discretized stochastic processes converge to the solution of the SDE. Moreover, using the fact that we know what the structure of the salary is as a function of the optimal control, we will also be able to construct an approximation of the optimal salary $S\left(u^{*}\right)$ by using $S\left(u_{n}\right)$. In other words, we will be able to give, with an arbitrary degree of accuracy, a fairly concrete presentation for the equilibrium strategies $\left(u^{*}, S^{*}\right)$ i.e. the control and the salary. As a by-product of our approach, we will show that the sequence is piecewise constant.

The paper is organized as follows. In section 2 we give an overview of the problem and we present our result. In section 3 we spell out the details and assumptions we need to prove our result. In section 4 we give a formal introduction to the problem we solve and we state our result in a formal way. In section 5 we set the stage for the discretization and in section 6 we prove the convergence results. In section 7 the reader will find a guide on how to apply

our results to concrete cases. It is important to stress that the application of this method is much simpler than the convergence proofs, which are necessary to show the validity of our approach, but are irrelevant for its application. In section 8 we outline how to use our approach to deal with a risk-neutral principal.

Finally, in Appendix I we discuss some technical issues and go deeper into the analysis of our assumptions. In particular, we show how the use of wellchosen transformations allows us to apply our result to a rather general class of situations. The longest proofs are in Appendix II.

\section{An Overview of the Continuous-Time Principal Agent Problem and the Equilibrium Strategies Characterization}

In this section we plan to give an overview of the results of agency theory in continuous-time we are going to use and, at the same time, explain our result 
in general terms. The mathematical assumptions that underpin our result are presented in the next section.

We investigate the optimal contract in a typical principal-agent model under moral hazard. At the beginning the principal and the agent agree on a certain salary $S$. This salary will depend on the outcome, which is the only common observable. We assume that the agent alone can control the output process, which is modelled with the following SDE

$$
d \mathbf{X}_{t}=f\left(t, \mathbf{X}_{t}, u_{t}\right) d t+\sigma\left(t, \mathbf{X}_{t}\right) d \mathbf{B}_{t}
$$

where $\mathbf{B}_{t}$ is a standard Brownian motion. As in any moral hazard problem, the effort or control is private information, so the principal does not know it. However, the principal can observe the output and can design and offer a salary which is a function of the output $S=S(\mathbf{X})$. Once the salary or contract has been offered, the agent will try, by using the available controls, to maximize

$$
\mathbf{E}\left[-\exp \left\{-r\left(S(\mathbf{X})-\int_{0}^{T} c\left(t, \mathbf{X}_{t}, u_{t}\right) d t\right)\right\}\right]
$$

(production is costly therefore $c$ ) while the principal will maximize

$$
\mathbf{E}[-\exp \{-R(F(\mathbf{X})-S(\mathbf{X}))\}] .
$$

In this context, by fully justifying the use of the first-order approach (see also [16] and [23]), Schaettler and Sung have been able to show that this game has an equilibrium where the agent receives a salary

$$
\begin{aligned}
S(\mathbf{X})= & \mathcal{W}_{0}+\int_{0}^{T} c\left(t, \mathbf{X}_{t}, u_{t}^{*}\right) d t+\int_{0}^{T} \nabla_{u} c\left(t, \mathbf{X}_{t}, u_{t}^{*}\right) D_{u}^{-1} f\left(t, \mathbf{X}_{t}, u_{t}^{*}\right) \sigma\left(t, \mathbf{X}_{t}\right) d \mathbf{B}_{t} \\
& +\frac{r}{2} \int_{0}^{T}\left\|\nabla_{u} c\left(t, \mathbf{X}_{t}, u_{t}^{*}\right) D_{u}^{-1} f\left(t, \mathbf{X}_{t}, u_{t}^{*}\right) \sigma\left(t, \mathbf{X}_{t}\right) d\right\|^{2} d t
\end{aligned}
$$

while the agent will supply the effort $u^{*}$. The analysis of Schaettler and Sung goes deeper as he shows that in the Markovian case the determination of the effort implies solving a PDE of type ${ }^{1}$

$$
\left\{\begin{array}{cc}
-\frac{\partial \mathcal{W}}{\partial t}+A(t) \mathcal{W}+Q(x, t, D \mathcal{W})=0 & x \in \mathbf{R}^{n}, t \in(0, T) \\
v(x, T)=F(x) & x \in \mathbf{R}^{n}
\end{array}\right.
$$

and this fully characterizes the solution. If we could solve this, we would know effort and, as a by-product, salary, but, in general, we cannot solve it. From the economic point-of-view this equation has a quite important part to play. In fact, the famous result of Holmström and Milgrom can be recovered easily from it. If we assume that the output is controlled by

$$
f(t, \mathbf{x}, \mathbf{u})=A(t) \mathbf{x}+B(t) \mathbf{u}
$$

\footnotetext{
1 More information about this equation will be provided later in the paper. At this point it is only necessary to point out that it is hard to solve.
} 
and the cost is quadratic $c=\mathbf{u}^{\mathrm{T}} M(t) \mathbf{u}$ we find that the solution of the game is given by

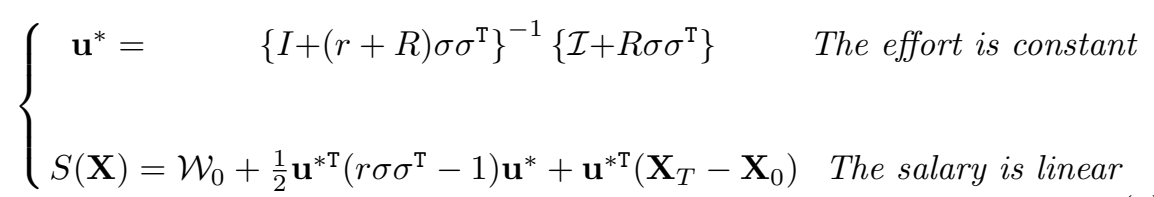

This is essentially the only known concrete result in the literature. The result shows that a very simple sharing rule can be obtained even though the agent has quite a considerable freedom in choosing strategies.

It is natural to ask what the contracts look like in general and if it is possible to find or compute something beyond this very special example. Suppose the output process is not a Brownian motion with drift. That is often reasonable. In many economic models we must use other processes (see [5]). We use geometric Brownian motion when we need to consider only positive quantities (for example, a manager in the financial industry with a portfolio and a shareholder). We use processes which exhibit mean reversion when we think that some sort of equilibrium level is an important feature of the problem (for example, a manager in the oil industry). We will construct approximations of the strategies at equilibrium by using very simple strategies. These strategies are locally constant and can be numerically computed.

\section{The Model}

In this section we present the model in a formal way and state our result. At the beginning the principal and the agent agree on a certain salary $S$. This salary will depend on the outcome, which is the only common observable. We assume that the agent alone can control the output process, which is modelled with a SDE.

In order to make our considerations and statements more precise, we need to give a few definitions and provide some clarifications about the model. If the reader feels that our description is little bit too terse, he/she should consult [33], where the same model is presented in a very detailed way

1. The information structure is represented by a filtration $\left\{\mathcal{F}_{t}: t \in[0, T]\right\}$ on a probability space $(\Omega, \mathcal{F}, \mathbf{P})$. There is a standard $n$-dimensional Brownian motion $\mathbf{B}=\left(B_{1}, \ldots, B_{n}\right)$ such that $B_{i}$ is independent of $B_{j}$ if $i \neq j$ and such that the variance of $B_{i}(t, \cdot)$ is $t$ and $B_{i}(t, \cdot)=\mathbf{E}\left(B_{i}(T, \cdot) \mid \mathcal{F}_{t}\right)$ and, if we denote with $\mathcal{N}$ the $\mathcal{F}$-null sets, we have

$$
\mathcal{F}_{t}=\sigma\left\{\mathbf{B}_{s} \mid 0 \leq s \leq t\right\} \cup \mathcal{N}
$$

So the Brownian motion generates the filtration we are using and the filtration has the standard properties. 
2. The principal and the agent agree at the start $(t=0)$ on a certain salary $S$, payable at time $T$. The salary is a function of a stochastic output process $\left\{\mathbf{X}_{t}\right\}$

$$
\mathbf{X}:[0, T] \times \Omega \rightarrow \mathbf{R}^{n}
$$

$S=S(\mathbf{X})$. This is the only common observable in the problem. Effort is not observable by the principal.

3. The output processes are given by (see [33] pp. 343 and 344) the following

$$
d \mathbf{X}_{t}=f\left(t, \mathbf{X}_{t}, u_{t}\right) d t+\sigma\left(t, \mathbf{X}_{t}\right) d \mathbf{B}_{t}
$$

and the processes are controlled by the agent (if we need to stress the control $u$ we write $\mathbf{X}_{t}^{u}$ instead of $\mathbf{X}_{t}$ ), via adapted controls

$$
\left\{u:[0, T] \times \Omega \rightarrow U \subset \mathbf{R}^{k} \mid u \text { is } \mathcal{F} \text { - adapted }\right\}
$$

where the set $U \subset \mathbf{R}^{k}$ is compact. We denote by $\mathcal{U}$ the set of all possible controls. We make the following assumptions:

i. The matrix valued function

$$
\sigma:[0, T] \times \Omega \rightarrow \mathbf{R}^{n \times n}
$$

has the following properties:

(a) each $\sigma_{i j}$ is $\mathcal{F}$-predictable;

(b) there is an $M_{1}$ such that $\left\|\sigma_{i j}(t, x)-\sigma_{i j}(t, y)\right\| \leq M_{1}\|x-y\|$;

(c) for each $(t, x)$ the matrix $\sigma(t, x)$ is invertible and there is a positive constant $M_{2}$ such that $\left\|\sigma(t, x)^{-1}\right\|<M_{2}$.

ii. The function

$$
f:[0, T] \times \Omega \times U \rightarrow \mathbf{R}^{n}
$$

has the following properties:

(a) for each $(t, x) f(t, x, \cdot): U \rightarrow \mathbf{R}^{n}$ is continuous;

(b) for each $u f(\cdot, \cdot, u):[0, T] \times \Omega \rightarrow \mathbf{R}^{n}$ is predictable;

(c) for each $(t, x, u),\|f(t, x, u)\|<M_{3}(1+\|x\|)$ for some positive constant $M_{3}$.

4. The agent has a discount rate $r$ and maximizes

$$
\mathbf{E}\left[-\exp \left\{-r\left(S(\mathbf{X})-\int_{0}^{T} c\left(t, \mathbf{X}_{t}, u_{t}\right) d t\right)\right\}\right] .
$$

We assume that

i. $c(t, x, u):[0, T] \times \mathbf{R}^{n} \times U \rightarrow \mathbf{R}$ is a continuous function;

ii. The cost

$$
c(t, x, u) \geq 0
$$

$\forall t \in[0, T], \forall x \in \mathbf{R}^{n}, \forall u \in U$

iii. $\left\|c\left(t, x_{1}, u\right)-c\left(t, x_{2}, u\right)\right\|_{\mathbf{R}^{n}} \leq K_{1}\left(1+\left\|x_{1}\right\|_{\mathbf{R}^{n}}+\left\|x_{2}\right\|_{\mathbf{R}^{n}}\right)\left\|x_{1}-x_{2}\right\|_{\mathbf{R}^{n}} \forall t \in$

$[0, T], \forall x_{1}, x_{2} \in \mathbf{R}^{n}, \forall u \in U$ for some positive constant $K_{1}$;

iv. $\|c(t, x, u)\|_{\mathbf{R}^{n}} \leq K_{1}\left(1+\|x\|_{\mathbf{R}^{n}}^{2}\right) \forall t \in[0, T], \forall x \in \mathbf{R}^{n}, \forall u \in U$. 
It must be noted that the last condition iv follows from condition iii. We have stated it explicitly since we will use it very often.

5. The principal has a discount rate $R$ and maximizes

$$
\mathbf{E}[-\exp \{-R(F(\mathbf{X})-S(\mathbf{X}))\}]
$$

If the principal maximizes his/her utility using, as constraint, the agent's first order condition we will say that she/he solves the relaxed problem.

As we have already written in the introduction our goal is to present, in the general case, a method to construct a sequence converging to the equilibrium strategies, by using piecewise linear controls. At this point we can be more clear and therefore we state our main result

Theorem 1 If $\left(u^{*}, S^{*}\right)$ is the pair of equilibrium strategies for the principalagent framework described above and $\mathbf{X}^{u^{*}}$ is the output produced at equilibrium, then there exists a sequence of controls $\left\{u_{n}\right\}$ and a sequence of discrete-time processes $\left\{\mathbf{X}^{u_{n}}\right\}$ such that:

1. For each $n \in \mathbf{N}, u_{n}$ is computable, solves the principal discretized relaxed problem, and the control is piecewise linear;

2. The sequence $\left\{u_{n}\right\}$ converges to $u^{*}$ with probability 1 ;

3. The sequence $\left\{S\left(\mathbf{X}^{u_{n}}\right)\right\}$ converges to $S^{*}$ in the $L^{2}$ norm;

4. The sequence $\left\{\mathbf{X}^{u_{n}}\right\}$ converges to the equilibrium output process $\mathbf{X}^{u^{*}}$ with probability 1.

\section{The Problem}

Using the fact that the principal cannot monitor the effort it is possible to show that the problem is equivalent to

$$
\left\{\begin{array}{cc}
\max _{u \in \mathcal{U}} & \mathbf{E}[-\exp -R(F(\mathbf{X})-S(\mathbf{X}))] \\
1 . & d \mathbf{X}_{t}^{u}=f\left(t, \mathbf{X}_{t}, u_{t}\right) d t+\sigma\left(t, \mathbf{X}_{t}\right) d \mathbf{B}_{t} \\
2 . & u \in \arg \max \mathbf{E}\left[-\exp \left\{-r\left(S(\mathbf{X})-\int_{0}^{T} c\left(t, \mathbf{X}_{t}, u_{t}\right) d t\right)\right\}\right] \\
3 . & \mathbf{E}\left[-e\left\{-r\left(S(\mathbf{X})-\int_{0}^{T} c\left(t, \mathbf{X}_{t}, u_{t}\right) d t\right)\right\}\right] \geq-\exp \left(-\mathcal{W}_{0}\right)
\end{array}\right.
$$

We know (see Schaettler and Sung's paper for more details) that the problem reduces to the following: the agent will receive the salary

$$
\begin{aligned}
S(\mathbf{X})= & \mathcal{W}_{0}+\int_{0}^{T} c\left(t, \mathbf{X}_{t}, u_{t}^{*}\right) d t+\int_{0}^{T} \nabla_{u} c\left(t, \mathbf{X}_{t}, u_{t}^{*}\right) D_{u}^{-1} f\left(t, \mathbf{X}_{t}, u_{t}^{*}\right) \sigma\left(t, \mathbf{X}_{t}\right) d \mathbf{B}_{t} \\
& +\frac{r}{2} \int_{0}^{T}\left\|\nabla_{u} c\left(t, \mathbf{X}_{t}, u_{t}^{*}\right) D_{u}^{-1} f\left(t, \mathbf{X}_{t}, u_{t}^{*}\right) \sigma\left(t, \mathbf{X}_{t}\right)\right\|^{2} d t
\end{aligned}
$$


where $u^{*}$ is the solution of the optimal control problem

$$
\left\{\begin{array}{ccc}
\max _{u \in \mathcal{U}} & \mathbf{E}[-\exp -R\{F(\mathbf{X})-S(\mathbf{X})\}] \\
d \mathbf{X}_{t}^{u} & =f\left(t, \mathbf{X}_{t}, u_{t}\right) d t+\sigma\left(t, \mathbf{X}_{t}\right) d \mathbf{B}_{t} \\
\mathbf{X}_{t}^{u} & = & x
\end{array}\right.
$$

and the solution gives, under certain conditions, the control at equilibrium. The principle of optimality gives

$$
\begin{aligned}
0= & \frac{\partial \mathcal{W}}{\partial t}(t, x)+\frac{1}{2} \operatorname{tr}\left\{\frac{\partial^{2} \mathcal{W}}{\partial x^{2}}(t, x) \sigma(t, x) \sigma^{\mathrm{T}}(t, x)\right\} \\
& -\frac{R}{2}\left\|\sigma^{T}(t, x) \frac{\partial^{2} \mathcal{W}}{\partial x^{2}}(t, x)\right\|^{2}+\max _{u \in U}\left\{\frac{\partial \mathcal{W}}{\partial t}(t, x)\right. \\
& \times\left\{f(t, x, u)+R \sigma\left(t, x_{t}\right) \sigma\left(t, x_{t}\right)^{\mathrm{T}}\left(\nabla_{u} c\left(t, x_{t}, u_{t}^{*}\right) D_{u}^{-1} f\left(t, x_{t}, u_{t}^{*}\right)\right)^{\mathrm{T}}\right\} \\
& \left.-\left(c(t, x, u)+\left(\frac{R+r}{2}\right)\left\|\left(\nabla_{u} c\left(t, x_{t}, u_{t}^{*}\right) D_{u}^{-1} f\left(t, x_{t}, u_{t}^{*}\right)\right)^{\mathrm{T}} \sigma\left(t, x_{t}\right)\right\|^{2}\right)\right\}
\end{aligned}
$$

While this equation is very useful from the heuristic point-of-view (see Schaettler and Sung's discussion) and in simple cases can be solved, it is otherwise not very useful to get information about the control itself, hence we cannot use it to learn about the strategies which are played at equilibrium. Such a nonlinear PDE is not very well understood. For this reason we will use an approach based entirely on Probability Theory which gives us a method to construct a solution for the problem above.

To start we prove, for the sake of completeness, the following well-known useful trick (see for example [33] and [9]) ${ }^{2}$

Lemma 1 The solution of the problem

$$
\left\{\begin{array}{ccc}
\max _{u \in \mathcal{U}} & \mathbf{E}_{\mathbf{P}}\left[\exp \left\{\int_{0}^{T} \ell\left(t, \mathbf{X}_{t}, u_{t}\right) d t+\Lambda_{1}\left(\mathbf{X}_{T}\right)+\Lambda_{2}(\mathbf{X})\right\}\right] \\
d \mathbf{X}_{t}^{u}= & g\left(t, \mathbf{X}_{t}, u\right) d t+\sigma\left(t, \mathbf{X}_{t}\right) d \mathbf{B}_{t} \\
\mathbf{X}_{0}^{u}= & x
\end{array}\right.
$$

where $\Lambda_{2}(\mathbf{X})=\int_{0}^{T} \varphi_{\Lambda}\left(t, \mathbf{X}_{t}, u_{t}\right) d \mathbf{B}_{t}^{u}$ with $\varphi_{\Lambda}$ bounded, is the same as the solution of the problem

$$
\left\{\begin{array}{ccc}
\max _{u \in \mathcal{U}} & \mathbf{E}_{\mathbf{P}_{u}}\left[\exp \left\{\int_{0}^{T} \ell\left(t, \mathbf{X}_{t}, u_{t}\right) d t+\Lambda_{1}\left(\mathbf{X}_{T}\right)\right\}\right] \\
d \mathbf{X}_{t}^{u} & =\left(g\left(t, \mathbf{X}_{t}^{u}, u_{t}\right)+\varphi_{\Lambda}\left(t, \mathbf{X}_{t}^{u}, u\right) \sigma\left(t, \mathbf{X}_{t}^{u}\right)\right) d t+\sigma\left(t, \mathbf{X}_{t}^{u}\right) d \mathbf{B}_{t} \\
\mathbf{X}_{0}^{u} & = & x
\end{array}\right.
$$

2 We assume, as usual, that $g$ satisfies $i i .(a)(b)(c)$. 
Proof Let

$$
I(u)=\mathbf{E}_{\mathbf{P}}\left[\exp \left\{\int_{0}^{T} \ell\left(t, \mathbf{X}_{t}, u\right) d t+\Lambda_{1}\left(\mathbf{X}_{T}\right)+\Lambda_{2}(\mathbf{X})\right\}\right]
$$

Since $\varphi_{\Lambda}$ is bounded,

$$
\Lambda_{2}(\mathbf{X})=\int_{0}^{T} \varphi_{\Lambda}\left(t, \mathbf{X}_{t}, u_{t}\right) d \mathbf{B}_{t}^{u}
$$

is an $L^{2}$ martingale. Thus

$$
\begin{aligned}
I(u)= & \mathbf{E}_{\mathbf{P}}\left[\exp \left\{\int_{0}^{T} \sigma^{-1} g\left(t, \mathbf{X}_{t}, u_{t}\right) d \mathbf{B}_{t}-\frac{1}{2} \int_{0}^{T}\left\|\sigma^{-1} g\left(t, \mathbf{X}_{t}, u_{t}\right)\right\|^{2} d t\right\}\right. \\
& \times \exp \left\{\Lambda_{1}\left(\mathbf{X}_{T}\right)+\int_{0}^{T}\left(\ell\left(t, \mathbf{X}_{t}, u_{t}\right)-\varphi_{\Lambda}\left(t, \mathbf{X}_{t}, u_{t}\right) \sigma^{-1} g\left(t, \mathbf{X}_{t}, u_{t}\right)\right) d t\right\} \\
& \left.\times \exp \left\{\int_{o}^{T} \varphi_{\Lambda}\left(t, \mathbf{X}_{t}, u_{t}\right) d \mathbf{B}_{t}\right\}\right]
\end{aligned}
$$

and therefore we can write

$$
\begin{aligned}
I(u)= & \mathbf{E}_{\mathbf{P}}\left[\exp \left\{\int_{0}^{T}\left(\sigma^{-1} g\left(t, \mathbf{X}_{t}, u_{t}\right)+\varphi_{\Lambda}\left(t, \mathbf{X}_{t}, u_{t}\right)\right) d \mathbf{B}_{t}\right\}\right. \\
& \times \exp \left\{-\frac{1}{2} \int_{0}^{T}\left\|\sigma^{-1} g\left(t, \mathbf{X}_{t}, u_{t}\right)+\varphi_{\Lambda}\left(t, \mathbf{X}_{t}, u_{t}\right)\right\|^{2} d t\right\} \\
& \left.\times \exp \left\{\Lambda_{1}\left(\mathbf{X}_{T}\right)+\int_{0}^{T}\left[\ell\left(t, \mathbf{X}_{t}, u_{t}\right)+\frac{1}{2}\left\|\varphi_{\Lambda}\left(t, \mathbf{X}_{t}, u_{t}\right)\right\|^{2}\right] d t\right\}\right]
\end{aligned}
$$

At this point we note that

$\mathbf{E}_{\mathbf{P}}\left[\exp \left\{\int_{0}^{T}\left(\sigma^{-1} g+\varphi_{\Lambda}\right)\left(t, \mathbf{X}_{t}, u_{t}\right)\right) d \mathbf{B}_{t}-\frac{1}{2} \int_{0}^{T}\left\|\left(\sigma^{-1} g+\varphi_{\Lambda}\right)\left(t, \mathbf{X}_{t}, u_{t}\right)\right\|^{2} d t\right\}$

defines a new probability measure. More precisely, it is known that there exists a probability measure $\mathbf{P}_{u}$, whose Radon-Nikodym derivative is given by

$$
\frac{d \mathbf{P}_{u}}{d \mathbf{P}}=\varphi_{u}
$$

where by definition

$$
\varphi_{u}=\mathbf{E}\left[\exp \left\{\int_{0}^{T}\left(\sigma^{-1} g+\varphi_{\Lambda}\right) d \mathbf{B}_{t}-\frac{1}{2} \int_{0}^{T}\left\|\left(\sigma^{-1} g+\varphi_{\Lambda}\right)\right\|^{2} d t\right\}\right]
$$

and by applying Girsanov's Theorem we obtain the desired result. 
Therefore, if we apply the last result to our framework (see [?]), we obtain the following useful simplification

Lemma 2 If we assume that $\nabla_{u} c(t, X, u) D_{u}^{-1} f\left(t, X_{t}, u_{t}\right) \sigma\left(t, X_{t}\right)$ is bounded then the principal's problem

$$
\left\{\begin{array}{rl}
\max _{u \in \mathcal{U}} & \mathbf{E}[-\exp -R\{F(\mathbf{X})-S(\mathbf{X})\}] \\
d \mathbf{X}_{t}^{u}= & f\left(t, \mathbf{X}_{t}, u_{t}\right) d t+\sigma\left(t, \mathbf{X}_{t}\right) d \mathbf{B}_{t} \\
\mathbf{X}_{0}^{u} & =
\end{array}\right.
$$

is equivalent to the following problem

$$
\left\{\begin{array}{ccc}
\max _{u \in \mathcal{U}} & \mathbf{E}\left[-\exp -R\left\{F(\mathbf{X})-\int_{0}^{T} c\left(t, \mathbf{X}_{t}, u_{t}\right) d t-\frac{r}{2} \int_{0}^{T}\left\|\left(\nabla_{u} c \cdot D_{u}^{-1} f\right) \sigma\left(t, \mathbf{X}_{t}\right)\right\|^{2} d t\right\}\right] \\
d \mathbf{X}_{t}^{u}= & \left(f\left(t, \mathbf{X}_{t}, u_{t}\right)+\left(\nabla_{u} c \cdot D_{u}^{-1} f\right)\left(t, \mathbf{X}_{t}, u_{t}\right) \sigma\left(t, \mathbf{X}_{t}\right)\right) d t+\sigma\left(t, \mathbf{X}_{t}\right) d \mathbf{B}_{t} \\
\mathbf{X}_{0}^{u}= & x
\end{array}\right.
$$

Proof Substitute

$$
\begin{aligned}
S(\mathbf{X})= & \mathcal{W}_{o}+\int_{0}^{T} c\left(t, \mathbf{X}_{t}, u_{t}\right) d t \\
& +\int_{0}^{T} \nabla_{u} c\left(t, \mathbf{X}_{t}, u_{t}\right) D_{u}^{-1} f\left(t, \mathbf{X}_{t}, u_{t}\right) \sigma\left(t, \mathbf{X}_{t}\right) d \mathbf{B}_{t} \\
& +\frac{r}{2} \int_{0}^{T}\left\|\nabla_{u} c\left(t, \mathbf{X}_{t}, u_{t}\right) D_{u}^{-1} f\left(t, \mathbf{X}_{t}, u_{t}\right) \sigma\left(t, \mathbf{X}_{t}\right)\right\|^{2} d t
\end{aligned}
$$

in the principal's maximand, and apply the previous lemma with

$$
\int_{0}^{T} \varphi_{\Lambda}(t, X, u) d \mathbf{B}_{t}=\int_{0}^{T} \nabla_{u} c\left(t, \mathbf{X}_{t}, u_{t}\right) D_{u}^{-1} f\left(t, \mathbf{X}_{t}, u_{t}\right) \sigma\left(t, \mathbf{X}_{t}\right) d \mathbf{B}_{t}
$$

In order to proceed we remind the reader (see [33]) that the principal maximizes

$$
\mathbf{E}[-\exp \{-R(F(\mathbf{X})-S(\mathbf{X}))\}]
$$

where we assume that the functional $F$ has the form ${ }^{3}$

$$
F(\mathbf{X})=F_{1}\left(\mathbf{X}_{T}\right)+\int_{0}^{T} a\left(t, \mathbf{X}_{t}\right) d t+\int_{0}^{T} b\left(t, \mathbf{X}_{t}, u_{t}\right) d \mathbf{B}_{t}
$$

where $F_{1}$ and $a\left(t, \mathbf{X}_{t}\right)$ and $b\left(t, \mathbf{X}_{t}\right)$ satisfy the following conditions

3 It is also common to write $F$ in this way

$$
F(\mathbf{X})=F_{1}\left(\mathbf{X}_{T}\right)+\int_{0}^{T} \alpha\left(t, \mathbf{X}_{t}\right) d t+\int_{0}^{T} \beta^{\mathrm{T}}\left(t, \mathbf{X}_{t}\right) d \mathbf{X}_{t},
$$

of course we have $a(t, x, u)=\alpha(t, x, u)+\beta^{\mathrm{T}}(t, x, u) f(t, x, u)$ and $b(t, x, u)=\beta^{\mathrm{T}}(t, x, u) \sigma(t, x)$ 
i. $\quad a(t, x):[0, T] \times \mathbf{R}^{n} \rightarrow \mathbf{R}$ is a continuous bounded function;

ii. $a(t, x, u)$ is not negative (i.e. $a(t, x, u) \geq 0$ ).

We also assume that

i. $b(t, x, u):[0, T] \times \mathbf{R}^{n} \times U \rightarrow \mathbf{R}^{n}$ is a continuous bounded function;

ii. the function $b(t, x, u) \sigma(t, x)$ is bounded.

We also assume that

i. $\quad F_{1}: \mathbf{R}^{n} \rightarrow \mathbf{R}$ is a continuous function bounded from below.

Remark 1 The reader should notice that the same strategy of Lemma 3 can be applied also to $F(X)$. In fact, since we assume that the functional $F$ can be written as

$$
F(\mathbf{X})=F_{1}\left(\mathbf{X}_{T}\right)+\int_{0}^{T} a\left(t, \mathbf{X}_{t}\right) d t+\int_{0}^{T} b\left(t, \mathbf{X}_{t}\right) d \mathbf{B}_{t}
$$

and we assume that $b\left(t, X_{t}\right)$, the volatility term, is reasonably tractable we can apply the last lemma. In this case we should replace the initial SDE with

$$
\begin{aligned}
d \mathbf{X}_{t}^{u}= & \left(f\left(t, \mathbf{X}_{t}, u_{t}\right)+\left(\left(\nabla_{u} c\left(\left(t, \mathbf{X}_{t}, u_{t}\right) D_{u}^{-1} f\left(t, \mathbf{X}_{t}, u_{t}\right)+b\left(t, \mathbf{X}_{t}\right)\right) \sigma\left(t, \mathbf{X}_{t}\right)\right) d t\right.\right. \\
& +\sigma\left(t, \mathbf{X}_{t}\right) d \mathbf{B}_{t}
\end{aligned}
$$

and proceed exactly as before (see Lemma 3).

To summarize, we maximize

$\mathbf{E}\left[-\exp -R\left\{F_{1}\left(\mathbf{X}_{T}\right)-\int_{0}^{T}\left(c\left(t, \mathbf{X}_{t}, u_{t}\right) d t+\frac{r}{2}\left\|\nabla_{u} c \cdot D_{u}^{-1} f\left(t, \mathbf{X}_{t}, u_{t}\right) \sigma\left(t, \mathbf{X}_{t}\right)\right\|^{2}-a\left(t, \mathbf{X}_{t}\right)\right) d t\right\}\right]$

using $u \in \mathcal{U}$, this means $u:[0, T] \times \Omega \rightarrow U \subset \mathbf{R}^{n}$ which is adapted, to control the process $\left\{\mathbf{X}_{t}^{u}\right\}_{t \in[0, T]}$.

The first thing to do is to construct a discrete version of this problem. This, of course, implies that we construct a discrete-time process which discretizes the process. Before we proceed it is useful to simplify the notation. Therefore, from this point on, we define $\psi\left(t, \mathbf{X}_{t}, u_{t}\right)$ as

$$
\begin{aligned}
\psi\left(t, \mathbf{X}_{t}, u_{t}\right)= & \left(\left(\nabla_{u} c\left(\left(t, \mathbf{X}_{t}, u_{t}\right) D_{u}^{-1} f\left(t, \mathbf{X}_{t}, u_{t}\right)+b\left(t, \mathbf{X}_{t}\right)\right) \sigma\left(t, \mathbf{X}_{t}\right)\right.\right. \\
& +f\left(t, \mathbf{X}_{t}, u_{t}\right)
\end{aligned}
$$

and we can re-write the process as

$$
d \mathbf{X}_{t}=\psi\left(t, \mathbf{X}_{t}, u_{t}\right) d t+\sigma\left(t, \mathbf{X}_{t}\right) d \mathbf{B}_{t}
$$

and, if we define

$$
\begin{aligned}
\widetilde{\mathbf{c}}\left(t, \mathbf{X}_{t}, u_{t}\right)= & -c\left(t, \mathbf{X}_{t}, u_{t}\right) d t \\
& -\frac{r}{2}\left\|\nabla_{u} c\left(t, \mathbf{X}_{t}, u_{t}\right) D_{u}^{-1} f\left(t, \mathbf{X}_{t}, u_{t}\right) \sigma\left(t, \mathbf{X}_{t}\right)\right\|^{2}+a\left(t, \mathbf{X}_{t}\right),
\end{aligned}
$$


we can re-write the problem in this way

$$
\left\{\begin{array}{ccc}
\max _{u \in \mathcal{U}} & \mathbf{E}\left[-\exp -R\left\{F_{1}\left(\mathbf{X}_{T}\right)+\int_{0}^{T} \widetilde{\mathbf{c}}\left(t, \mathbf{X}_{t}, u_{t}\right) d t\right\}\right] \\
d \mathbf{X}_{t}^{u}= & \psi\left(t, \mathbf{X}_{t}, u_{t}\right) d t+\sigma\left(t, \mathbf{X}_{t}\right) d \mathbf{B}_{t} \\
\mathbf{X}_{0}^{u}= & x
\end{array}\right.
$$

from now on we will refer to this problem as the $(\mathcal{P} \mathcal{A})$ problem or just $(\mathcal{P} \mathcal{A})$. It is possible to show that if we define

$$
\left\{\begin{array}{l}
\varphi_{\mathbf{X}, u}(t, \omega)=\exp -R\left\{F_{1}\left(\mathbf{X}_{T}\right)+\int_{t}^{T} \widetilde{\mathbf{c}}\left(s, \mathbf{X}_{t}^{u}, u_{t}\right) d s\right\} \\
\mathcal{J}(t, \mathbf{X}, u)=\quad \mathbf{E}_{t, x} \exp \left\{\varphi_{\mathbf{X}, u}(t, \omega)\right\}
\end{array}\right.
$$

and the associated value function

$$
\inf _{u \in U} \mathcal{J}(0, \mathbf{X}, u)=\exp -R v(x, 0)
$$

then $v(x, t)$ is the solution of the following

$$
\left\{\begin{array}{cl}
-\frac{\partial v}{\partial t}+A(t) v+Q(x, t, D v)=0 & x \in \mathbf{R}^{n}, t \in(0, T) \\
v(x, T)=F_{1}(x) & x \in \mathbf{R}^{n}
\end{array}\right.
$$

where the operators appearing in the equations are defined as

$$
A(t) z=-\operatorname{tr}\left(\mathbf{a}(x, t) D^{2} z\right) \quad \text { with } \quad \mathbf{a}=1 / 2 \cdot \sigma \sigma^{\mathrm{T}}
$$

and

$$
Q(x, t, p)=-\left\{-\operatorname{Ra}(x, t) p^{\prime} \cdot p+\inf _{u \in U}(\widetilde{\mathbf{c}}(t, \mathbf{x}, u)+\psi(t, \mathbf{x}, u) \cdot p)\right\}
$$

This is a quasi-linear parabolic type of equation. It is clearly very hard to solve and very little is known about the properties of the solution.

\section{The Discretization}

In the last section we explained why it is not possible to solve the problem by using the PDE Bellman Equation. We are thus left with the problem of how to approximate the strategies at equilibrium. The approach that we use is to replace the original process with a discrete time process (for a different type of procedure see [30]). In the second step we discretize the control accordingly and we solve the discrete problem, producing a sequence of simple controls. Of course, we need to prove that the limit behaves well. Therefore, we proceed in this way (see [19]): in this section we first discretize the process, before producing the sequence of simple controls. We then postpone the proofs of convergence to the next section, where we will also make clear (i.e. we give 
the appropriate topologies) what kind of convergence is to be proved (for a different type of procedure see the very interesting [3] and [1] and [30]).

To give the characterization we start with the SDE governing the output process,

$$
d \mathbf{X}_{t}=\psi\left(t, \mathbf{X}_{t}, u_{t}\right) d t+\sigma\left(t, \mathbf{X}_{t}\right) d \mathbf{B}_{t}
$$

By Ito's Formula,

$$
\left.\mathcal{L}^{u} g\left(\mathbf{X}_{t}\right)=g\left(\mathbf{X}_{0}\right)\right)+\int_{0}^{t}\left(\mathcal{L}^{u(s)} g\right)\left(\mathbf{X}_{s}\right) d s+\int_{0}^{t} g_{x}^{\prime}\left(\mathbf{X}_{s}\right) \sigma\left(\mathbf{X}_{s}\right) d \mathbf{B}_{s}
$$

where

$$
\begin{aligned}
\mathcal{L}^{u} g(x) & =g_{x}^{\prime}(x) \psi(x, u)+\frac{1}{2} \operatorname{tr}\left[g_{x x} \sigma \sigma^{\mathrm{T}}(t, x)\right] \\
& =\sum_{j=1}^{n} \psi_{j}(x, u) \frac{\partial g}{\partial x_{j}}+\frac{1}{2} \sum_{j, i=1}^{n} a_{i j}(x, u) \frac{\partial^{2} g}{\partial x_{j} \partial x_{i}}
\end{aligned}
$$

If we define

$$
W(x, t)=\mathbf{E}_{x}\left[-\exp -R\left\{F_{1}\left(\mathbf{X}_{T}\right)+\int_{t}^{T} \widetilde{\mathbf{c}}\left(s, \mathbf{X}_{s}, u_{s}\right) d s\right\}\right]
$$

we have

$$
\frac{\partial W}{\partial t}(x, t, u)+\mathcal{L}^{u} W(x, t, u)-\widetilde{\mathbf{c}} W(x, t, u)=0
$$

or, in more explicit fashion,

$$
\frac{\partial W}{\partial t}+\sum_{j=1}^{n} \psi_{j}(x, u) \frac{\partial W}{\partial x_{j}}(x, t, u)+\frac{1}{2} \sum_{j, i=1}^{n} a_{i j}(x, u) \frac{\partial^{2} W}{\partial x_{j} \partial x_{j}}(x, t, u)-R \widetilde{\mathbf{c}} W(x, t, u)=0
$$

This PDE will be the starting point for our discretization process. Before we proceed we want to emphasize one major point: that we do not need to know what the solution of this equation is. Rather, we shall interpret it in a formal way and use it as a guide to discretize the output process under examination. After we fix $\delta$ and $h$ bigger than zero such that $T / \delta$ is an integer we consider the grid $G_{h, \delta}=\left\{(x+\ell h, n \delta) \mid \ell \in \mathbf{Z}_{h}\right.$ and $\left.n=0,1, \ldots, T / \delta\right\}$ and, given a problem $(\mathrm{P})$

$$
\left\{\begin{array}{ccc}
\max _{u \in \mathcal{U}} & \mathbf{E}\left[-\exp -R\left\{\widehat{F}\left(\mathbf{X}_{T}\right)+\int_{0}^{T} \widehat{\mathbf{c}}\left(t, \mathbf{X}_{t}, u_{t}\right) d t\right\}\right] \\
d \mathbf{X}_{t}^{u}= & \widehat{\psi}\left(\mathbf{X}_{t}, u_{t}\right) d t+\widehat{\sigma}\left(\mathbf{X}_{t}\right) d \mathbf{B}_{t} \\
\mathbf{X}_{t}^{u}= & \mathbf{x}
\end{array}\right.
$$


we say that the process $(\xi, \omega)$, defined on the grid $G_{h, \delta}$, is a locally consistent discretization of $(\mathrm{P})$ if

$$
\left\{\begin{array}{c}
\mathbf{E} \Delta \xi_{n}=\widehat{\psi}\left(\xi_{n}, \omega_{n}\right) \delta+o(\delta) \\
\operatorname{cov} \Delta \xi_{n}=\widehat{\sigma} \widehat{\sigma}^{\mathrm{T}} \delta+o(\delta)
\end{array}\right.
$$

and $\omega$ maximizes $\mathbf{E}\left[-\exp -R\left\{\widehat{F}(\xi)+\sum \widehat{\mathbf{c}}\left(n, \xi_{n}, \omega_{n}\right) \delta\right\}\right]$.

The basic idea is to study our problem with a discrete process substituted for the solution of the SDE and to use the equation above to define the transition probabilities as a function of the control. We proceed in this way because we want that the discretization, which depends on $\delta$ and $h$, looks like the solution of the initial SDE when the parameters go to zero. In order to achieve this it is sufficient to prove that the discretization is locally consistent.

It is important to stress that for the moment we do not concern ourselves with convergence, but rather we focus our attention on the discrete process and we study the mean and the variance of the discretized process as functions of the spatial discretization parameter $\delta$. This is done in the

Theorem 2 If $\widehat{F}, \widehat{\mathbf{c}}, \widehat{\psi}$, and $\widehat{\sigma}$ are bounded and continuous then $(P)$ has a locally consistent discretization.

\section{Proof See Appendix II.}

The method applied to prove this theorem is based on a careful choice of the discretization we use for the partial derivatives that appear in the PDE. It turns out that, after we choose the discretization in the correct way and we re-write the PDE, the coefficients of the resulting discrete equation can be used as the transition probabilities that we need in order to construct the discrete controlled output produced by the agent.

In order to proceed we need to give the following

Definition 1 A function $\chi_{B\left(\mathbf{R}^{n}\right)}^{m}: \mathbf{R}^{n} \rightarrow \mathbf{R}^{n}$ is called a cut-off function if

$$
\chi_{B\left(\mathbf{R}^{n}\right)}^{m}(x)=\left\{\begin{array}{cr}
x & \text { if }\|x\|_{\mathbf{R}^{n}} \leq m \\
l_{m}\left(\|x\|_{\mathbf{R}^{n}}\right)\left(x /\|x\|_{\mathbf{R}^{n}}\right) & \text { if }\|x\|_{\mathbf{R}^{n}}>m
\end{array}\right.
$$

where $l_{m}:[m,+\infty) \rightarrow \mathbf{R}_{+}$is an increasing differentiable function such that $l_{m}(x)=m$ when $\|x\|_{\mathbf{R}^{n}}=m$ and $l_{m}\left(\|x\|_{\mathbf{R}^{n}}\right) \leq N_{m} \forall x \in \mathbf{R}^{n} \backslash m B\left(\mathbf{R}^{n}\right)$ where $N_{m}$ is finite, $N_{m}>m$ and $m B\left(\mathbf{R}^{n}\right)=\left\{x \in \mathbf{R}^{n}\|x\|_{\mathbf{R}^{n}}<m\right\}$.

We now state an important consequence of the previous theorem 
Theorem 3 The problem $(P A)_{m}$

$$
\left\{\begin{array}{ccc}
\max _{u \in \mathcal{U}} & \mathbf{E}\left[-\exp -R\left\{\chi_{B\left(\mathbf{R}^{n}\right)}^{m} \circ F_{1}\left(\mathbf{X}_{T}\right)+\int_{0}^{T} \chi_{B\left(\mathbf{R}^{n}\right)}^{m} \circ \widetilde{\mathbf{c}}\left(t, \mathbf{X}_{t}, u_{t}\right) d t\right\}\right] \\
d \mathbf{X}_{t}^{u}= & \chi_{B\left(\mathbf{R}^{n}\right)}^{m} \circ \psi\left(t, \mathbf{X}_{t}, u_{t}\right) d t+\chi_{B\left(\mathbf{R}^{n}\right)}^{m} \circ \sigma\left(\mathbf{X}_{t}\right) d \mathbf{B}_{t} \\
\mathbf{X}_{t}^{u} & = & x
\end{array},\right.
$$

where $\chi_{B(\mathbf{R})}^{m}$ is a cut-off function, has a discretization in the grid $G_{h, \delta}$ which is locally consistent.

Proof Since $\chi_{B(\mathbf{R})}^{m} \circ F_{1}, \chi_{B(\mathbf{R})}^{m} \circ \widetilde{\mathbf{c}}, \chi_{B\left(\mathbf{R}^{n}\right)}^{m} \circ \psi$, and $\chi_{B\left(\mathbf{R}^{n}\right)}^{m} \circ \sigma\left(\mathbf{X}_{t}\right)$ are bounded we can apply the previous theorem.

Finally we notice that

Remark 2 Given a sequence $\left\{\delta_{n}\right\}$ with $\delta_{n} \rightarrow 0$ and a sequence $\left\{h_{n}\right\}$ with $h_{n} \rightarrow$ 0 (with $\delta_{n} / 2 h_{n}^{2} \rightarrow 0$ ) then, for the problem $(P A)_{m}$, it is possible to construct on the grids $G_{h_{n}, \delta_{n}}$ two sequences of processes: the stochastic controls $\left\{u_{m, n}\right\}$ and the locally consistent discrete output processes $\left\{\mathbf{X}_{m}^{h_{n}, \delta_{n}}\right\}$ sequence. In fact, we can apply the last theorem for any $n$ since the boundedness condition is always satisfied for the problem $(P A)_{m}$.

\section{Convergence Proofs}

In this section we are going to show that the sequences we constructed in the last section converge to equilibrium strategies, thus proving our main result. To prove this convergence result we need to set the stage in order to talk about convergence and then we need to prove three facts:

i. the sequence of the discrete stochastic processes converges to the solution of the SDE

$$
d \mathbf{X}_{t}=\psi\left(t, \mathbf{X}_{t}, u_{t}\right) d t+\sigma\left(t, \mathbf{X}_{t}\right) d \mathbf{B}_{t}
$$

ii. the sequence of optimal controls for the discrete optimal control problems is convergent;

iii. the limit of the sequence of optimal control for the discrete problem is an optimal control for the continuous time problem.

The method is based on the notion of weak convergence for probability measures. In order to make more intelligible what follows, we briefly summarize the most important aspects of this notion. Of course there are excellent books on this topic ([2]) and we encourage the reader to look at them for an in-depth discussion of this notion.

In general, given any metric space $\left(M, d_{M}\right)$, we denote with the symbol $B_{M}$ the Borel sigma algebra generated by the metric topology, i.e. the minimal sigma 
algebra which contains every open set of the topology induced by the metric, and we denote with the symbol $P(M)$ the space of probability measures on $\left((M, d), \mathcal{B}_{M}\right)$. On this space it is possible to define a new metric, called the Prohorov metric, in this way

$$
\pi\left(\mathbf{P}_{1}, \mathbf{P}_{2}\right)=\inf \left\{\epsilon>0 \mid \mathbf{P}_{1}(C) \leq \mathbf{P}_{2}(C)+\epsilon \forall C \in \mathcal{B}_{M} \quad \text { closed }\right\} .
$$

The notion is very important and it is possible to prove that if $\left(M, d_{M}\right)$ is separable and complete then $(P(M), \pi)$ is also complete and separable. If $\left\{\mathbf{P}_{\alpha}, \alpha \in A\right\}$ is a family of elements of $(P(M), \pi)$ we say that the family is tight if for each $\epsilon>0$ there is a compact set $K_{\epsilon} \subseteq M$ such that

$$
\inf _{\alpha \in A} P_{\alpha}\left(K_{\epsilon}\right) \geq 1-\epsilon
$$

It is a well-known and a very important fact for us, due to Prohorov, that a family $\left\{\mathbf{P}_{\beta}, \beta \in B\right\}$ has a compact closure in $(P(M), \pi)$ if and only if $\left\{\mathbf{P}_{\beta}, \beta \in B\right\}$ is tight.

It is also possible to introduce a similar notion for random variables. The idea is rather simple. Let us assume that we have a sequence of random variables, which are not necessarily defined on the same space,

$$
X_{n}:\left(\Omega_{n}, \mathcal{F}_{n}, \mathbf{Q}_{n}\right) \rightarrow(M, d) \quad n \in \mathbf{N}
$$

We say that they converge weakly to $X$, and we write $X_{n} \Rightarrow X$, if and only if

$$
\mathbf{E}_{n} h\left(X_{n}\right) \rightarrow \mathbf{E} h(X)
$$

for any $h \in C_{b}(M, d)$. In other words, we have $X_{n} \Rightarrow X$ if and only

$$
\int_{M} h(s) \mathbf{Q}_{n}(d s) \rightarrow \int_{M} h(s) \mathbf{Q}(d s)
$$

$\forall h \in C_{b}(M, d)=\{f:(M, d) \rightarrow \mathbf{R} \mid f$ is continuous and bounded $\}$.

In what follows we will study a problem by modifying the data via cut-off functions. In order to simplify the notation we adopt a simple convention. If we start with the problem $(\mathcal{P} \mathcal{A})$ and a sequence of cutoff functions $\left\{\chi_{B\left(\mathbf{R}^{n}\right)}^{m}\right\}$ then we will denote with the symbol $(\mathcal{P} \mathcal{A})_{m}$ the problem that we obtain if we compose any function appearing in $(\mathcal{P} \mathcal{A})$ with the cut-off function $\chi_{B\left(\mathrm{R}^{n}\right)}^{m}$; we shall refer to this problem as the $m$-truncated version of $(\mathcal{P} \mathcal{A})$. In what follows we shall use the cut-off functions to modify the problem at hand. In fact, we will use the functions to create problems with bounded data and give fairly concrete descriptions of the problems with bounded data. This means that we will show that with such a problem it is possible to construct a piecewise constant control and piecewise constant output process that can approximate the solution of the cutoff problem arbitrarily closely. Given the discussion above, and the definition of $\widetilde{\mathbf{c}}$ and the construction of $\left\{\mathbf{X}_{m}^{h_{n}, \delta_{n}}\right\}$, the reader should not be surprised that we need to assume, as in Schaettler and Sung's paper, the implementability condition (see pp. 352 and Theorem 4.2 in [33]) and the following: 
A1 The optimal effort is unique;

A2 $F_{1}$ is bounded below;

A3 The functions $\left\|\nabla_{u} \chi_{B\left(\mathbf{R}^{n}\right)}^{m} \circ c(t, x, u) D_{u}^{-1} \chi_{B\left(\mathbf{R}^{n}\right)}^{m} \circ f(t, x, u) \chi_{B\left(\mathbf{R}^{n}\right)}^{m} \circ \sigma(t, x)\right\|$ and $\left\|\nabla_{u} c(t, x, u) D_{u}^{-1} f(t, x, u) \sigma(t, x)\right\|$ are bounded and continuous.

A4 We assume that the problem under investigation is regular in the sense that if we take a sequence of cutoff functions $\left\{\chi_{B\left(\mathbf{R}^{n}\right)}^{m}\right\}$ then the solution $\left(\mathbf{X}_{t}^{m}, u_{t}^{m}\right)$ of the same problem with output given by

$$
\left\{\begin{array}{lll}
d \mathbf{X}_{t}^{m}= & \left(\chi_{B\left(\mathbf{R}^{n}\right)}^{m} \circ \psi\left(t, \mathbf{X}_{t}^{m}, u_{t}\right)\right) d t+\chi_{B\left(\mathbf{R}^{n}\right)}^{m} \circ \sigma\left(\mathbf{X}_{t}^{m}\right) d \mathbf{B}_{t} & \\
\mathbf{X}_{\tau}^{m}=x & \forall t \in[\tau, T]
\end{array}\right.
$$

converges, as $m \rightarrow \infty$, to the solution of the original problem and there exists a sequence of sets $\left\{A_{m}\right\}$ and a constant $B$ such that $\mathbf{P}\left(A_{m}\right) \rightarrow 1$ and for every $(\omega, t) \in A_{m} \times[0, T] \mathbf{X}_{t}^{m}(\omega, t)=\mathbf{X}_{t}(\omega, t)$ and $\mathbf{1}_{A_{n}^{c}} \int_{0}^{T}\left|\mathbf{X}_{t}^{m}-\mathbf{X}_{t}\right|^{2} \leq B$ a.e. on $\Omega{ }^{4}$

Remark 3 The reader should notice that this property is only about existence and continuity, with respect to truncation, of the solution. It does not really say anything about the construction of the optimal control. It is fortunate that this property is actually easily checked in many situations and is satisfied by many problems we are dealing with (see Appendix I). For instance, the property holds trivially for the Holmström and Milgrom case, in fact we assume that $U$ is a compact, therefore bounded set.

In Appendix I the reader will find more about this, the assumptions $(\mathbf{F})$, $(\mathbf{C}),(\mathbf{G})$ and $(\mathbf{H})$ and the proof of the following

Theorem 4 Given the model $(\mathcal{P} \mathcal{A})$ described at the beginning, if there exists an open set $\mathcal{O}$ such that $\mathbf{X}_{t} \in \mathcal{O} \subseteq \mathbf{R}^{n}$ and for all $y \in \mathcal{O} \sigma$ is non-singular with the inverse matrix satisfying the following condition

$$
\exists \mathcal{G} \in \mathcal{C}^{2}\left(\mathcal{O}, \mathbf{R}^{n}\right) \quad \mathcal{G}_{y}(y)=\sigma^{-1} \quad \forall y \in \mathcal{O}
$$

and $F_{1} \circ G^{-1}, \widetilde{\mathbf{c}}\left(t, G^{-1}, u_{t}\right)$ and the drift term of $G\left(\mathbf{X}_{t}\right)$ satisfy the assumptions $(\mathbf{F}),(\mathbf{C}),(\mathbf{G})$ and $(\mathbf{H})$ then the solution of the $m$-truncated problem $(\mathcal{P} \mathcal{A})_{m}$ converges to the solution of $(\mathcal{P} \mathcal{A})$.

Proof See Appendix I.

Remark 4 (About the Structure of Theorem 10) Essentially, by using a transformation $G$ and Ito's formula we reduce the problem to the case where we have a process with constant stochastic volatility. This case is not trivial but

\footnotetext{
4 The reader should notice that this is quite natural since it is possible to prove, even in a more general setting, that if we define $Y_{n}^{2}=\sup _{[0, T]}\left|\mathbf{X}_{t}^{n}-\mathbf{X}_{t}\right|^{2}$ then (see [10]) $\mathbf{E} Y_{n} \rightarrow 0$ so we can conclude $Y_{n} \rightarrow 0$ in probability.
} 
it is easier to prove that the problem has the property A4. We stress that the main idea behind this result is again to exploit the possibility of the interplay between the objective function and the SDE. In this case the interplay is a little bit more complex but the basic idea is very simple. In order to give an intuition of our strategy we would like to point out that $(\mathcal{P} \mathcal{A})$ is equivalent to solving the following (notice that we minimize)

$$
\left\{\begin{array}{ccc}
\min _{u \in \mathcal{U}} & \mathbf{E}\left[\exp -R\left\{F_{1}\left(\mathbf{X}_{T}\right)+\int_{0}^{T} \widetilde{\mathbf{c}}\left(t, \mathbf{X}_{t}, u_{t}\right) d t\right\}\right] \\
d \mathbf{X}_{t}^{u}= & \psi\left(t, \mathbf{X}_{t}, u_{t}\right) d t+\sigma\left(t, \mathbf{X}_{t}\right) d \mathbf{B}_{t} \\
\mathbf{X}_{0}^{u}= & x
\end{array}\right.
$$

and since $\log$ is monotone this, in turn, is equivalent to solving

$$
\left\{\begin{array}{ccc}
\min _{u \in \mathcal{U}} & \log \mathbf{E}\left[\exp -R\left\{F_{1}\left(\mathbf{X}_{T}\right)+\int_{0}^{T} \widetilde{\mathbf{c}}\left(t, \mathbf{X}_{t}, u_{t}\right) d t\right\}\right] \\
d \mathbf{X}_{t}^{u} & = & \psi\left(t, \mathbf{X}_{t}, u_{t}\right) d t+\sigma\left(t, \mathbf{X}_{t}\right) d \mathbf{B}_{t} \\
\mathbf{X}_{0}^{u} & = & x
\end{array}\right.
$$

If we assume that there exists an open set $\mathcal{O}$ such that $\mathbf{X}_{t} \in \mathcal{O} \subseteq \mathbf{R}^{n}$ and for all $y \in \mathcal{O} \sigma$ is non-singular with the inverse matrix satisfying the following condition $\exists G \in \mathcal{C}\left(\mathcal{O}, \mathbf{R}^{n}\right) G_{y}(y)=\sigma^{-1} \forall y \in \mathcal{O}$, then we can transform, by using Ito's Formula, the initial problem in the following equivalent problem

$$
\left\{\begin{array}{ccc}
\min _{u \in \mathcal{U}} & \log \mathbf{E}\left[\exp -R\left\{F_{1}\left(G^{-1}\left(\mathbf{Y}_{T}\right)\right)+\int_{0}^{T} \widetilde{\mathbf{c}}\left(t, G^{-1}\left(\mathbf{Y}_{t}\right), u_{t}\right) d t\right\}\right] \\
d \mathbf{Y}_{t}^{u}= & \bar{\psi}\left(t, \mathbf{Y}_{t}, u_{t}\right) d t+d \mathbf{B}_{t} \\
\mathbf{Y}_{0}^{u}= & G(x)
\end{array}\right.
$$

where $\bar{\psi}\left(t, \mathbf{Y}_{t}, u_{t}\right) d t+d \mathbf{B}_{t}$ is uniquely determined by applying Ito's Formula to $G\left(\mathbf{X}_{t}\right)$. In the first Appendix we will develop a strategy to show that the solutions of the truncated versions of $(\mathcal{P})$ converge to the solution of $(\mathcal{P})$ and therefore $\mathbf{A} 4$ is satisfied. We stress that this is not simple and it will require us to establish several auxiliary results. In order to ensure this convergence we need to be sure that the objective functions and the equations satisfy some regularity conditions which are the conditions $((\mathbf{F}),(\mathbf{C}),(\mathbf{G})$ and $(\mathbf{H})$ named above and we spell them out in detail in the beginning of the first Appendix.

Remark 5 While the proof of the stated theorem is quite long and a little complex it is quite easy to explain what the Theorem is useful for. In fact, let us assume we are working with $d \mathbf{X}_{t}=f\left(t, \mathbf{X}_{t}, u_{t}\right) d t+\sigma\left(\mathbf{X}_{t}\right) d \mathbf{B}_{t}$. If we take any differentiable function $G$ we have, for $Y_{t}=G\left(\mathbf{X}_{t}\right)$, the following

$$
d \mathbf{Y}_{t}=\left(\frac{d G}{d x}\left(\mathbf{X}_{t}, u_{t}\right)+\frac{1}{2} \frac{d^{2} G}{d x^{2}} \sigma\left(\mathbf{X}_{t}\right)^{2}\right) d t+\frac{d G}{d x} \sigma\left(\mathbf{X}_{t}\right) d \mathbf{B}_{t}
$$


if we can find an increasing differential $G$ such that $\frac{d G}{d x} \sigma(x)=1$ then we can rewrite

$$
\mathbf{Y}_{t}=\widehat{b}\left(\mathbf{Y}_{t}, u\right) d t+d \mathbf{B}_{t}
$$

and this equation is easier to handle than the original one since it has constant volatility. The Theorem has some quite pleasant consequences. For example, let us think of a model where the initial output is driven, via controls, by a geometric Brownian motion and we have a quadratic cost function. We can observe that the function $G(y)=\log y$ would satisfy the hypothesis so we can conclude that a model with a GBM and quadratic cost is in our range.

In this section we prove convergence of the discrete process to the original one. Namely, in the proposition in the last section we proved that the discrete version of the problem can be solved and this, via the notion of local consistency, gives us a discrete time Markov chain $\left\{\mathbf{X}_{m}^{h_{n}, \delta_{n}}\right\}$ and a sequence of controls which are step functions $\left\{u_{m, n}\right\}$.

We can construct a new process (see [19]), which is the continuous-time interpolation of the process $\left\{\mathbf{X}_{m, n}^{h}\right\}$. It is possible to show that the process is given by

$$
\varphi^{h}(t)=x+\int_{o}^{t} b\left(\varphi^{h}(s), u^{h}(s)\right) d s+M^{h}(t)+\delta_{1}^{h}(t)
$$

where $M^{h}(t)$ is a martingale and its quadratic variation is given by

$$
\left[M^{h}\right]_{t}=\int_{0}^{t} a\left(\varphi^{h}(s)\right) d s+\delta_{2}^{h}(t) \quad \text { where } \sup _{s \leq t} \mathbf{E}\left|\delta_{2}^{h}(s)\right| \rightarrow 0
$$

It is possible to prove that there exists a Brownian Motion $\mathbf{B}_{n}^{h}$ such that we can write

$$
M^{h}(t)=x+\int_{o}^{t} \sigma\left(\varphi^{h}(s)\right) d \mathbf{B}^{h}+\epsilon_{1}^{h}(t)
$$

where, for each $t$, we have $\sup _{s<t} \mathbf{E}\left|\epsilon_{1}^{h}(s)\right| \rightarrow 0$ as $h \rightarrow 0$.

At this point, we note that we can apply the results of Chapter 10 and Chapter 12 of [19] in order to account for the boundary condition. We also note that in our case the applications of the results are very simple since we are working with the simplifying assumption that the jump-component of the process is zero (i.e. $\left.\int_{0}^{t} \int_{-} q(\mathbf{X}(s-)) \mathbf{N}(d s d \rho)=0\right)$. Therefore, we get the following

Theorem 5 In the $m$-truncated model $(\mathcal{P} \mathcal{A})_{m}$ let $\left\{\mathbf{X}_{m}^{h_{n}, \delta_{n}}\right\}$ the approximating chain be locally consistent with the initial process. Let $\left\{u_{m, n}\right\}$ denote the admissible sequence of controls which is used. Let $\left\{\varphi_{m, n}\right\}$ denote the continuous parameter interpolation of the chain and Re $u_{m}^{h}$ a relaxed control representation of $\left\{u_{m, n}\right\}$ for the chain $\left\{\varphi_{m, n}^{h}\right\}$ and let $\left\{\tau_{m, n}^{h}\right\}$ be a family of stopping times. Then $\left(\left\{\varphi_{m, n}^{h}\right\},\left\{u_{m, n}^{h}\right\},\left\{\mathbf{B}^{h}\right\}\right.$, Re $\left.u_{m}^{h}\right)$ is tight. Let $\left(\mathbf{X}_{m}, u_{m}, \mathbf{B}, \tau\right)$ be 
the limit and $\left\{\mathcal{F}_{t}\right\}_{t \in[0, T]}$ the sigma algebra induced by $\left(\mathbf{X}_{m}, u_{m}, \mathbf{B}, \tau\right)$, then $\mathbf{B}$ is a Brownian motion, $\tau$ is a $\left\{\mathcal{F}_{t}\right\}$ stopping time and we have

$\mathbf{X}_{m, t}=x+\int_{0}^{t} \int_{\mathcal{U}}\left(\chi_{B\left(\mathbf{R}^{n}\right)}^{m} \circ \psi\left(\mathbf{X}_{m, s}, \alpha\right)\right) \operatorname{Re} u_{m}^{h}(d \alpha) d s+\int_{0}^{t} \chi_{B\left(\mathbf{R}^{n}\right)}^{m} \circ \sigma\left(\mathbf{X}_{m, s}\right) d \mathbf{B}_{s}$.

in other words the limit satisfies the initial truncated SDE.

The most important step in order to give the proof of the main Theorem has just been completed. In fact, we also notice (see [19]) that the boundedness of $\chi_{B\left(\mathbf{R}^{n}\right)}^{m} \circ \psi, \chi_{B\left(\mathbf{R}^{n}\right)}^{m} \circ \sigma, \chi_{B\left(\mathbf{R}^{n}\right)}^{m} \circ F, \chi_{B\left(\mathbf{R}^{n}\right)}^{m} \circ c$ together with the use of Fatou's Lemma and Skorokhod's Representation Theorem and the last theorem imply that the sequences $\left\{\mathbf{X}_{m}^{h_{n}, \delta_{n}}\right\}$ and $\left\{u_{m}^{h_{n}, \delta_{n}}\right\}$, which converge to $\mathbf{X}_{m}$ and $u_{m}$, are maximizing for the problem $(\mathcal{P} \mathcal{A})_{m}$.

The reader should see that to prove the rest is quite simple now that we are in a position to use the machinery of weak convergence. We just remind the reader that the defining property of weak convergence is that: $X_{n} \Rightarrow X$ if and only if

$$
\mathbf{E}_{n} g\left(X_{n}\right) \rightarrow \mathbf{E} g(X)
$$

for any $g$ bounded and continuous.

We now prove the convergence for the cost and the salary function.

Theorem 6 If we define

$$
X_{n}=\exp \left[-R\left\{F_{1}\left(\mathbf{X}_{n}^{h_{n}, \delta_{n}}\right)+\int_{0}^{T} \widetilde{c}\left(t, \mathbf{X}_{n}^{h_{n}, \delta_{n}}, u_{n}^{h_{n}, \delta_{n}}\right) d t\right\}\right]
$$

then

$$
\lim _{n \rightarrow \infty} \mathbf{E} X_{n}=\mathbf{E}\left[-\exp \left[-R\left\{F_{1}\left(\mathbf{X}_{T}\right)+\int_{0}^{T} \widetilde{c}\left(t, \mathbf{X}_{t}, u_{t}\right) d t\right\}\right]\right]
$$

holds.

\section{Proof See Appendix II}

The intuition behind this result is quite simple. In fact, since the process $\left\{\mathbf{X}_{n}^{h_{n}, \delta_{n}}, u_{n}^{h_{n}, \delta_{n}}\right\}$ is the solution of the discretized truncated problem then it has good convergence properties (as a consequence of the weak convergence) and therefore it converges to the continuous version of the truncated problem. In order to establish the result we need to use our assumptions about the function $c$. In other words, we would like to stress that given our assumptions $\mathrm{A} 1, \mathrm{~A} 2$ and A3 we need only to study the difference $\chi_{B\left(\mathbf{R}^{n}\right)}^{n} \circ c\left(t, \mathbf{X}_{t}^{n}, u_{t}^{n}\right)-$ $c\left(t, \mathbf{X}_{t}, u_{t}\right)$. In the proof we need only to study this difference very carefully to be sure that the right condition are in place in order to apply Lebesgue's Dominated Convergence Theorem. This is equivalent to showing the convergence of a series, which is done in the final part of the proof.

We also observe that 
Remark 6 There is a way to relax the assumptions we used to prove the Theorem, which is of help in specific cases. If we could find a function $\gamma(x)$ such that:

1. $\lim _{x \rightarrow \infty} \gamma(x) / x=\infty$;

2. $\sup _{n} \mathbf{E}\left[\gamma\left(-\exp -R\left\{F_{1}\left(\mathbf{X}_{T}^{n}\right)+\int_{0}^{T} \widetilde{\mathbf{c}}\left(t, \mathbf{X}_{t}^{n}, u_{t}^{n}\right) d t\right\}\right)\right]=B<\infty$ the conclusion would hold. In fact, if we define

$$
X_{n}=\exp -R\left\{F_{1}\left(\mathbf{X}_{T}^{n}\right)+\int_{0}^{T} \widetilde{\mathbf{c}}\left(t, \mathbf{X}_{t}^{n}, u_{t}^{n}\right) d t\right\}
$$

and set $\phi(x)=\gamma(x) / x$, we have the following inequality

$$
\mathbf{E}\left[X_{n} \mathbf{1}\left(X_{n} \geq x\right)\right]=\mathbf{E}\left[\frac{\phi(x)}{\gamma(x)} \mathbf{1}\left(X_{n} \geq x\right)\right] \leq \frac{\sup \mathbf{E}\left[\gamma\left(X_{n}\right)\right]}{\min _{z \geq x} \gamma(x)}
$$

Using the last inequality we deduce that

$$
\lim _{x \rightarrow \infty} \mathbf{E}\left[X_{n} \mathbf{1}\left(X_{n} \geq x\right)\right]=0
$$

In fact, we have

$$
\lim _{x \rightarrow \infty} \mathbf{E}\left[X_{n} \mathbf{1}\left(X_{n} \geq x\right)\right]=\lim _{x \rightarrow \infty}\left[\frac{\phi(x)}{\gamma(x)} \mathbf{1}\left(X_{n} \geq x\right)\right] \leq \lim _{x \rightarrow \infty} \frac{B}{\min _{x \geq z} \gamma(z)}
$$

We conclude that the family

$$
X_{n}=\exp -R\left\{F_{1}\left(\mathbf{X}_{T}^{n}\right)+\int_{0}^{T} \widetilde{\mathbf{c}}\left(t, \mathbf{X}_{t}^{n}, u_{t}^{n}\right) d t\right\}
$$

is uniformly integrable, implying that

$$
\mathbf{E}\left[-\exp -R\left\{F_{1}\left(\mathbf{X}_{T}^{n}\right)+\int_{0}^{T} \widetilde{\mathbf{c}}\left(t, \mathbf{X}_{t}^{n}, u_{t}^{n}\right) d t d t\right\}\right]
$$

converges to

$$
\mathbf{E}\left[-\exp -R\left\{F_{1}\left(\mathbf{X}_{T}\right)+\int_{0}^{T} \widetilde{\mathbf{c}}\left(t, \mathbf{X}_{t}, u_{t}\right) d t\right\}\right]
$$

and this completes the proof.

Theorem 7 If we define

$$
\begin{aligned}
S_{n}(\mathbf{X})= & \mathcal{W}_{o}+\int_{0}^{T} c\left(\mathbf{X}_{n}^{h_{n}, \delta_{n}}, u_{n}^{h_{n}, \delta_{n}}\right) d t \\
& +\int_{0}^{T} \nabla_{u} c\left(\mathbf{X}_{n}^{h_{n}, \delta_{n}}, u_{n}^{h_{n}, \delta_{n}}\right) D_{u}^{-1} f\left(\mathbf{X}_{n}^{h_{n}, \delta_{n}}, u_{n}^{h_{n}, \delta_{n}}\right) \sigma\left(\mathbf{X}_{n}^{h_{n}, \delta_{n}}\right) d \mathbf{B}_{t} \\
& +\frac{r}{2} \int_{0}^{T}\left\|\nabla_{u} c\left(\mathbf{X}_{n}^{h_{n}, \delta_{n}}, u_{n}^{h_{n}, \delta_{n}}\right) D_{u}^{-1} f\left(\mathbf{X}_{n}^{h_{n}, \delta_{n}}, u_{n}^{h_{n}, \delta_{n}}\right) \sigma\left(\mathbf{X}_{n}^{h_{n}, \delta_{n}}\right)\right\|^{2} d t
\end{aligned}
$$


then

$$
\lim _{n \rightarrow \infty} S_{n}(\mathbf{X})=S(\mathbf{X}) \text { in } L^{2} .
$$

Proof See Appendix II.

The intuition behind this result is also quite simple but more work is necessary since we need to establish convergence in the $L^{2}$ norm. For this reason we need to treat in a different way the last two elements of the contract. Of course, an important role is played by the fact that the process $\left\{\mathbf{X}_{n}^{h_{n}, \delta_{n}}, u_{n}^{h_{n}, \delta_{n}}\right\}$ is the solution of the discretized truncated problem therefore it has good convergence properties (as consequence of the weak convergence) and this allows us to obtain the convergence for the last two elements of the contract quite readily. In order to establish the result we need also to use our assumptions about the function $c$ and the fact that the moments of solutions of SDE can be easily estimated. In fact, we only need to use in a very efficient way the standard inequalities (Cauchy-Schwarz) and the assumption A4 and the Dominated Convergence Theorem.

We also observe that as a consequence we obtain

Theorem $8\left\{S_{n}(\mathbf{X})\right\}$ converges to $S(\mathbf{X})$ in probability.

Finally, if we use the results of this section we have

Theorem 9 If $\left(u^{*}, S^{*}\right)$ is the pair of equilibrium strategies for the principalagent framework described above and $\mathbf{X}^{u^{*}}$ is the output produced at equilibrium, then there exists a sequence of controls $\left\{u_{n}\right\}$ and a sequence of discrete-time processes $\left\{\mathbf{X}^{u_{n}}\right\}$ such that:

1. For each $n \in \mathbf{N}, u_{n}$ is computable, solves the principal discretized relaxed problem, and the control is piecewise linear;

2. The sequence $\left\{u_{n}\right\}$ converges to $u^{*}$ with probability 1 ;

3. The sequence $\left\{S\left(\mathbf{X}^{u_{n}}\right)\right\}$ converges to $S^{*}$ in the $L^{2}$ norm;

4. The sequence $\left\{\mathbf{X}^{u_{n}}\right\}$ converges to the equilibrium output process $\mathbf{X}^{u^{*}}$ with probability 1.

\section{Recipes for Contracts}

At this point we explain how it is possible to use the proved results in order to construct the approximations for the efforts, salaries and outputs process. To simplify the description we assume that the output process is a scalar and there is only one Brownian motion. We denote the initial condition for the SDE $d \mathbf{X}_{t}=\Psi_{f, c, \sigma} d t+\sigma d \mathbf{B}_{t}$ governing the output process as $x_{0}$. We consider the set $G_{h, \delta}=\left\{\left(x_{0}+\ell h, n \delta\right) \mid \ell \in \mathbf{Z}\right.$ and $\left.n=0, \ldots, T / \delta\right\}$ where $T$ is finite and, for a suitable $\delta>0, N_{\delta_{n}}=T / \delta$ is a positive integer and $h>0$. The reader should think of this set as the production possibilities of an agent who can change production but only making discrete changes at discrete time.

The basic idea is to study our problem while we substitute the solution of the SDE with a discrete process with states in $G_{h, \delta}$. Since the states are in 
the grid we need only to specify the transition probabilities. In other words, given that the current state at time $t=\ell \delta$ is $y=x_{0}+m h$ we need to give the transition probability $p(y, y+h \mid u), p(y, y-h \mid u), p(y, y \mid u)$ for the possible states $y+h$ and $y-h$ and $y$ at time $t+\delta=(\ell+1) \delta$.

The problem of finding the transition probabilities can be solved by observing that by discretizing the objective function we obtain the following function

$$
\mathbf{E}\left[-\exp -R\left\{-\sum \chi_{B(\mathbf{R})}^{m} \circ \widetilde{\mathbf{c}}_{f, c, \sigma}\left(x_{n \delta}, u\right) \delta+\chi_{B(\mathbf{R})}^{m} \circ F\left(x_{N_{\delta} \delta}\right)\right\}\right]
$$

and by observing that there is a solution for the maximization problem. Let $u: G_{h, \delta} \rightarrow U$ be the solution of the finite maximization problem (1)

$$
\max _{U} \mathbf{E}\left[-\exp -R\left\{-\sum \chi_{B(\mathbf{R})}^{m} \circ \widetilde{\mathbf{c}}_{f, c, \sigma}\left(x_{n \delta}, u\right) \delta+\chi_{B(\mathbf{R})}^{m} \circ F\left(x_{N_{\delta} \delta}\right)\right\}\right]
$$

Then we define transition probabilities in the following way (2):

for the transition from $y$ to $y+h$ we set

$$
p(y, y+h \mid u)=\frac{\delta}{2 h^{2}} \sigma(y)+\Psi_{f, c, \sigma}^{+}\left((y, u(y, \ell)) \frac{\delta}{h}\right.
$$

for the transition from $y$ to $y-h$ we set

$$
p(y, y-h \mid u)=\frac{\delta}{2 h^{2}} \sigma(y)+\Psi_{f, c, \sigma}^{-}\left((y, u(y, \ell)) \frac{\delta}{h}\right.
$$

for the transition from $y$ to $y$ we set

$$
p(y, y \mid u)=1-\frac{\delta}{2 h^{2}} \sigma(y)-\left|\Psi_{f, c, \sigma ; j}(y, u(y, \ell))\right| \frac{\delta}{h}
$$

Therefore, given $\delta>0$ and $h$, we can construct, by solving a finite dimensional optimization problem, a discrete process $\mathbf{X}^{u}$.

If we fix a sequence of $\delta_{n}$ such that $\delta_{n} \rightarrow 0$ and $h_{n}\left(\right.$ with $\delta_{n} / 2 h_{n}^{2} \rightarrow 0$ ) then we are able to construct two sequences of processes: the stochastic controls $\left\{u_{n}\right\}$ sequence and the discrete output processes $\left\{\mathbf{X}^{u_{n}}\right\}$ sequence. We also note that we can re-write the approximate salaries in this way (3)

$$
\begin{aligned}
S\left(\mathbf{X}^{u_{n}}\right)= & \mathcal{W}_{0}+\sum_{\ell=0}^{N_{\delta_{n}}-1} c\left(\mathbf{X}_{\ell \delta}^{u_{n}}, u_{n, \ell \delta}\right) \delta_{n}+ \\
& \sum_{\ell=0}^{N_{\delta_{n}}-1} c^{\prime}\left(\mathbf{X}_{t}^{u_{n}}, u_{n, \ell \delta}\right) D_{u}^{-1} f\left(\mathbf{X}_{\ell \delta}^{u_{n}}, u_{n, \ell \delta}\right) \sigma\left(\mathbf{X}_{\ell \delta}^{u_{n}}\right) \\
& \times\left(\frac{\left(\mathbf{X}_{(\ell+1) \delta}^{u_{n}}-\mathbf{X}_{\ell \delta}^{u_{n}}\right)-\Psi_{f, c, \sigma}\left(\mathbf{X}_{\ell \delta}^{u_{n}}, u_{n, \ell \delta}\right) \delta_{n}}{\sigma\left(\mathbf{X}_{\ell \delta}^{u_{n}}\right)}\right) \\
& +\frac{r}{2} \sum_{\ell=0}^{N_{\delta_{n}-1}}\left\|c^{\prime}\left(\mathbf{X}_{t}^{u_{n}}, u_{n, \ell \delta}\right) D_{u}^{-1} f\left(\mathbf{X}_{\ell \delta}^{u_{n}}, u_{n, \ell \delta}\right) \sigma\left(\mathbf{X}_{\ell \delta}^{u_{n}}\right)\right\|^{2} \delta_{n}
\end{aligned}
$$


where, as we said, $N_{\delta_{n}}=T / \delta_{n}$.

It is important to notice that the procedure does not involve the use of the Brownian Motion since this can be recovered (see section 6) intrinsically from the production process itself. Moreover, it is important to underline that after we solve the maximization problem (1) then it is straightforward to construct the output (2) and the salary (3) and therefore we do not need to solve any PDE which is extremely complex (see section 4 for more about this).

\section{The Risk-Neutral Principal}

In this section we draft briefly how to apply our results to the case when the principal is risk-neutral. In this case the salary would be the same as in the CARA case (see Holmstrom's paper)

$$
\begin{aligned}
S(\mathbf{X})= & \mathcal{W}_{0}+\int_{0}^{T} c\left(\mathbf{X}_{t}, u_{t}^{*}\right) d t+\int_{0}^{T} \nabla_{u} c\left(\mathbf{X}_{t}, u_{t}^{*}\right) D_{u}^{-1} f\left(\mathbf{X}_{t}, u_{t}^{*}\right) \sigma\left(\mathbf{X}_{t}\right) d \mathbf{B}_{t} \\
& +\frac{r}{2} \int_{0}^{T}\left\|\nabla_{u} c\left(\mathbf{X}_{t}, u_{t}^{*}\right) D_{u}^{-1} f\left(\mathbf{X}_{t}, u_{t}^{*}\right) \sigma\left(\mathbf{X}_{t}\right)\right\|^{2} d t
\end{aligned}
$$

where the optimal control $u^{*}$ is the solution of the optimal control problem, solved by the principal

$$
\left\{\begin{array}{ccc}
\max _{u \in \mathcal{U}} & \mathbf{E}[\{F(\mathbf{X})-S(\mathbf{X})\}] \\
d \mathbf{X}_{t}^{u} & =f\left(t, \mathbf{X}_{t}, u_{t}\right) d t+\sigma\left(t, \mathbf{X}_{t}\right) d \mathbf{B}_{t} \\
\mathbf{X}_{t}^{u} & = & \mathbf{x}
\end{array}\right.
$$

We do not need to apply the transformation of measure proved in section 2. Instead, we should write down the PDE that is relevant for the linear case. If the process is given by

$$
d \mathbf{X}_{t}=f\left(\mathbf{X}_{t}, u_{t}\right) d t+\sigma\left(\mathbf{X}_{t}\right) d \mathbf{B}_{t}
$$

and if we define

$$
\mathcal{L}^{u}=\sum_{j=1}^{n} f_{j}(x, u) \frac{\partial}{\partial x_{j}}+\frac{1}{2} \sum_{j, i=1}^{n} a_{i j}(x, u) \frac{\partial^{2}}{\partial x_{j} \partial x_{i}}
$$

then we have, by Ito's Formula,

$$
\mathcal{L}^{u} g(X(t))=g(X(0))+\int_{0}^{t}\left(\mathcal{L}^{u(s)} g\right)(X(s)) d s+\int_{0}^{t} g_{x}^{\prime}(X(s)) \sigma(X(s)) d \mathbf{B}_{s}
$$


where

$$
\begin{aligned}
\mathcal{L}^{u} g(x) & =g_{x}^{\prime}(x) f(x, u)+\frac{1}{2} \operatorname{tr}\left[g_{x x} \sigma \sigma^{\mathrm{T}}(t, x)(t, x)\right] \\
& =\sum_{j=1}^{n} f_{j}(x, u) \frac{\partial g}{\partial x_{j}}+\frac{1}{2} \sum_{j, i=1}^{n} a_{i j}(x, u) \frac{\partial^{2} g}{\partial x_{j} \partial x_{i}}
\end{aligned}
$$

It is possible to show that if we define

$$
W(x, t)=\mathbf{E}_{x}[F(\mathbf{X})-S(\mathbf{X})]
$$

then we have

$$
\mathcal{L}^{u} W(x, t)-\widetilde{\mathbf{c}}=0
$$

or, in more explicit fashion,

$$
\sum_{j=1}^{n} f_{j}(x, u) \frac{\partial W}{\partial x_{j}}(x, t)+\frac{1}{2} \sum_{j, i=1}^{n} a_{i j}(x, u) \frac{\partial^{2} W}{\partial x_{j} \partial x_{j}}(x, t)-\widetilde{\mathbf{c}}=0
$$

At this point it is possible to find a discretization procedure as we did in the CARA case and then to find the approximation scheme. Local consistency and the rest are not difficult and the convergence proofs are exactly as in the previous case.

\section{Appendix I}

The goal of this section is to prove Theorem 10. As we mentioned earlier, the proof is not very short so we present it here. In the first part of the Appendix we solve a simpler problem and then, with the help of Ito's Formula, we will show that this is actually general enough for our purpose.

The goal of this section is really to adapt well-known constructions and proofs to our setting. We also need to use a logarithmic transform. For this reason we are going to minimize $\mathbf{E} \exp y$ rather than maximizing $\mathbf{E}[-\exp y$ ], of course the two are equivalent.

Let us start with

$$
\left\{\begin{array}{ccc}
\min _{u \in \mathcal{U}} & \log \mathbf{E}\left[\exp \left\{\int_{0}^{T} \ell\left(t, \mathbf{X}_{t}, u_{t}\right) d t+G_{1}\left(\mathbf{X}_{T}\right)\right\}\right] \\
d \mathbf{X}_{t}= & f\left(t, \mathbf{X}_{t}, u\right) d t+\sigma d \mathbf{B}_{t} \\
\mathbf{X}_{s}= & x
\end{array}\right.
$$

where we make the following assumptions on the data:

(F)

F i. $\quad f(t, x, u):[0, T] \times \mathbf{R}^{n} \times U \rightarrow \mathbf{R}$ is a continuous function; 
F ii. $\forall u \in U$ and $\forall t \in[0, T] f(\cdot, \cdot, u):[0, T] \times \Omega \rightarrow \mathbf{R}^{d}$ is $\mathcal{F}_{t}$-predictable in $(t, x)$;

F iii. $\|f(u, x, t)\|_{\mathbf{R}^{n}} \leq K\left(1+\|x\|_{\mathbf{R}^{n}}\right) \quad \forall u \in U, \forall t \in[0, T], \forall x \in \mathbf{R}^{n}$

and $(\mathbf{C})$

C i. $\ell(t, x, u):[0, T] \times \mathbf{R}^{n} \times U \rightarrow \mathbf{R}$ is a continuous function;

C ii. there is a positive constant $M_{1}$ such that

$$
\ell(t, x, u) \geq-M_{1}
$$

$\forall t \in[0, T], \forall x \in \mathbf{R}^{n}, \forall u \in U$

C iii. $\left\|\ell\left(t, x_{1}, u\right)-\ell\left(t, x_{2}, u\right)\right\|_{\mathbf{R}^{n}} \leq K_{1}\left(1+\left\|x_{1}\right\|_{\mathbf{R}^{n}}+\left\|x_{2}\right\|_{\mathbf{R}^{n}}\right)\left\|x_{1}-x_{2}\right\|_{\mathbf{R}^{n}} \forall t \in$ $[0, T], \forall x \in \mathbf{R}^{n}, \forall u \in U$.

C iv. $\left\|\ell\left(t, x_{1}, u\right)\right\|_{\mathbf{R}^{n}} \leq K_{1}\left(1+\left\|x_{1}\right\|_{\mathbf{R}^{n}}^{2}\right) \forall t \in[0, T], \forall x \in \mathbf{R}^{n}, \forall u \in U$

and $(\mathbf{G})$

$\mathbf{G}$ i. $\quad G_{1}: \mathbf{R}^{n} \rightarrow \mathbf{R}$ is a continuous function;

$\mathbf{G}$ ii. there is a positive constant $N_{1}$ such that

$$
G_{1}(x) \geq-N_{1}
$$

$\forall x \in \mathbf{R}^{n}$.

G iii. $\left\|G_{1}\left(x_{1}\right)-G_{1}\left(x_{2}\right)\right\|_{\mathbf{R}^{n}} \leq K_{2}\left(1+\left\|x_{1}\right\|_{\mathbf{R}^{n}}+\left\|x_{2}\right\|_{\mathbf{R}^{n}}\right)\left\|x_{1}-x_{2}\right\|_{\mathbf{R}^{n}} \quad \forall x \in$ $\mathbf{R}^{n}$.

G iv. $\left\|G_{1}(x)\right\|_{\mathbf{R}^{n}} \leq K_{2}\left(1+\|x\|_{\mathbf{R}^{n}}^{2}\right) \forall x \in \mathbf{R}^{n}$

At this point it is necessary to say a few words about the SDE we are going to use. The reader has probably already noticed that we are working with a very special class of SDE. To make things very clear let us fix a probability space $(\Omega, \mathcal{F}, \mathbf{P})$ and on it we take an $n$-dimensional Brownian motion $\mathbf{B}=\left(B_{1}, \ldots B_{n}\right)$ with its natural filtration $\left\{\mathcal{F}_{t}: t \in[0, T]\right\}$ such that $B_{i}$ is independent of $B_{j}$ if $i \neq j$ and such that the variance of $B_{i}(t, \cdot)$ is $t$ and $B_{i}(t, \cdot)=\mathbf{E}\left(B_{i}(T, \cdot) \mid \mathcal{F}_{t}\right)$. The information structure is represented by the filtration $\left\{\mathcal{F}_{t}: t \in[0, T]\right\}$. Let us denote with the symbol $U \subset \mathbf{R}^{k}$ a compact set. Given a positive $\sigma>0$ we consider the following class of SDE's

$$
d Y_{t}=g\left(t, Y_{t}, u\right) d t+\sigma d \mathbf{B}_{t} \quad \text { with } \quad Y_{0}=y \in \mathbf{R}^{n}
$$

(notice that we use $\sigma$ to also denote $\sigma I$ where $I$ is the $n \times n$ identity matrix.) where $u \in \mathcal{U}$ and, by definition,

$$
\mathcal{U}=\{u:[0, T] \times \Omega \rightarrow U: u \text { is } \mathcal{F} \text { - adapted }\}
$$

We say that the SDE has a strong solution if there exists a process $Y=$ $\left\{Y_{t}\right\}_{t \in[0, T]}$ such that

1. $Y$ is $\mathcal{F}_{t}$ - adapted;

2. $P\left(Y_{0}=y\right)=1$; 
3. For any $j$ and any $t \in[0, T] \mathbf{P}\left(\int_{0}^{t}\left|g_{j}\left(t, Y_{t}, u_{t}\right)\right| d t<\infty\right)=1$;

4. The SDE is satisfied for any $t \in[0, T]$.

It is well-known that an equation such as $d Y_{t}=g\left(t, Y_{t}, u\right) d t+\sigma d \mathbf{B}_{t}$ with $Y_{0}=y \in \mathbf{R}^{n}$ has always a strong solution as long $\sigma$ is a positive constant. Since we use only this type of SDE we do not need to use anything more than what we have just stated.

In order to simplify we are going to introduce a few definitions. To study the problem we introduce the following functions

$$
\left\{\begin{array}{l}
\varphi_{x, u}(s, \omega)=\int_{s}^{T} \ell\left(t, \mathbf{X}_{t}, u_{t}\right) d t+G_{1}\left(\mathbf{X}_{T}\right) \\
\mathcal{J}(s, x, u)=\mathbf{E}_{s, x} \exp \left\{\varphi_{x, u}(s, \omega)\right\}
\end{array}\right.
$$

We define, as usual, the following

$$
V(s, x)=\inf _{u \in U} \log \mathcal{J}(s, x, u)
$$

and

$$
\Psi(s, x)=\inf _{u \in U} \mathcal{J}(s, x, u) .
$$

We use the symbol $\mathbf{X}_{t}^{m}$ to denote the solution of the SDE

$$
\left\{\begin{array}{l}
d \mathbf{X}_{t}^{m}=\left(\chi_{B\left(\mathbf{R}^{n}\right)}^{m} \circ f\right)\left(t, \mathbf{X}_{t}^{m}, u\right) d t+\sigma d \mathbf{B}_{t} \\
\mathbf{X}_{s}^{m}=x
\end{array} \quad \forall t \in[s, T]\right.
$$

What follows is a standard argument to prove the existence of the control with obvious modifications to fit our setting. If $v, w \in \mathbf{R}^{n}$ we will use the symbol $\langle v, w\rangle_{\mathbf{R}^{n}}$ to denote the inner product, if there is not any possible confusion we will use $\langle v, w\rangle$ instead of $\langle v, w\rangle_{\mathbf{R}^{n}}$ and $|v|$ instead of $\|v\|_{\mathbf{R}^{n}}$.

Lemma 3 If we define

$$
\Psi_{m}=\inf _{u \in U} \mathbf{E}_{s, x} \exp \left\{\int_{s}^{T} \ell\left(t, \mathbf{X}_{t}^{m}, u_{t}\right) d t+G_{1}\left(\mathbf{X}_{T}^{m}\right)\right\}
$$

where

$$
d \mathbf{X}_{t}^{m}=\chi_{B\left(\mathbf{R}^{n}\right)}^{m} \circ f\left(t, \mathbf{X}_{t}^{m}, u\right) d t+\sigma d \mathbf{B}_{t}
$$

the following are true:

i. The function $\Psi_{m}$ is in $C^{1,2}$ and it satisfies the following

$$
\left\{\begin{array}{ccc}
\frac{\partial \Psi_{m}}{\partial \tau}+\frac{1}{2} \sigma^{2} \Delta \Psi_{m} & =-\min _{u \in U}\left[\left\langle\chi_{B\left(\mathbf{R}^{n}\right)}^{m} \circ f(s, x, u), \nabla \Psi_{m}\right\rangle+\chi_{B\left(\mathbf{R}^{n}\right)}^{m} \circ \ell(s, x, u) \Psi_{m}\right] \\
\Psi_{m}(T, x) & = & \exp G_{1}(x)
\end{array}\right.
$$


ii. There is an admissible control $u^{*}$ such that

$$
\Psi_{m}(s, x)=\mathcal{J}\left(s, x, u^{*}\right)
$$

i.e. the inf exists.

Proof To start we note that the equation has a solution since it is a consequence of Theorem 5.8.1 in [21] (p. 495 and p. 496), moreover the solution is a bounded solution in the $C^{1,2}$ class (Theorem 5.3.1 in [21], p. 437). Since the solution of the SDE has a $L^{2}$ martingale part we apply Ito's Formula and we obtain the following formula

$$
\begin{aligned}
\Psi_{m}\left(t, \mathbf{X}_{t}^{m}\right)= & \Psi_{m}(s, x)+\sigma \int_{s}^{t} \nabla \Psi_{m}\left(\tau, \mathbf{X}_{\tau}^{m}\right) d \mathbf{B}_{\tau}+ \\
& +\int_{s}^{t}\left(\frac{\partial \Psi_{m}}{\partial \tau}\left(\tau, \mathbf{X}_{\tau}^{m}\right)+\left\langle\nabla \Psi_{m}\left(\tau, \mathbf{X}_{\tau}^{m}\right), \chi_{B\left(\mathbf{R}^{n}\right)}^{m} \circ f(t, x, u)\right\rangle\right) d \tau \\
& +\int_{s}^{t} \frac{\sigma^{2}}{2} \Delta \Psi_{m}\left(\tau, \mathbf{X}_{\tau}^{m}\right) d \tau
\end{aligned}
$$

and, since

$$
\mathbf{M}_{t}=\exp \int_{s}^{t}\left(\chi_{B\left(\mathbf{R}^{n}\right)}^{m} \circ \ell\left(\tau, \mathbf{X}_{\tau}^{m}, u_{\tau}\right)\right) d \tau
$$

is a continuous semimartingale, we have

$$
\begin{aligned}
\mathbf{M}_{t} \Psi_{m}\left(t, \mathbf{X}_{t}^{m}\right)= & \mathbf{M}_{s} \Psi_{m}(s, x)+\int_{s}^{t} \mathbf{M}_{\tau} \Psi_{m}\left(\tau, \mathbf{X}_{\tau}^{m}\right)\left(\chi_{B\left(\mathbf{R}^{n}\right)}^{m} \circ \ell\right)\left(\tau, \mathbf{X}_{\tau}^{m}, u\right) d \tau+ \\
& \int_{s}^{t} \mathbf{M}_{\tau}\left(\frac{\partial \Psi_{m}}{\partial \tau}\left(\tau, \mathbf{X}_{\tau}^{m}\right)+\left\langle\nabla \Psi_{m}\left(\tau, \mathbf{X}_{\tau}^{m}\right), \chi_{B\left(\mathbf{R}^{n}\right)}^{m} \circ f(\tau, x, u)\right\rangle\right) d \tau+ \\
& \sigma^{2} \int_{s}^{t} \mathbf{M}_{\tau} \nabla \Psi_{m}\left(\tau, \mathbf{X}_{\tau}^{m}\right) d \mathbf{B}_{\tau}+\frac{\sigma^{2}}{2} \int_{s}^{t} \mathbf{M}_{\tau} \Delta \Psi_{m}\left(\tau, \mathbf{X}_{\tau}^{m}\right) d \tau
\end{aligned}
$$

and this implies that $\Psi_{m}(s, x) \leq E_{s, x} \mathbf{M}_{T} \Psi_{m}\left(T, \mathbf{X}_{T}^{m}\right)$ and therefore, by using the fact that $\Psi_{m}$ is the solution of the equation

$$
\left\{\begin{array}{ccc}
\frac{\partial \Psi_{m}}{\partial \tau}+\frac{1}{2} \sigma^{2} \Delta \Psi_{m} & =-\min _{u \in U}\left[\left\langle\chi_{B\left(\mathbf{R}^{n}\right)}^{m} \circ f(s, x, u), \nabla \Psi_{m}\right\rangle+\chi_{B\left(\mathbf{R}^{n}\right)}^{m} \circ \ell(s, x, u) \Psi_{m}\right] \\
\Psi_{m}(T, x) & = & \exp G_{1}(x)
\end{array}\right.
$$


and taking $t=T$, we can conclude that $\Psi_{m}(s, x) \leq J\left(s, x, u^{*}\right)$. Since the minimization problem

$$
\arg \min _{u \in U}\left[\left\langle\chi_{B\left(\mathbf{R}^{n}\right)}^{m} \circ f(s, x, u), \nabla \Psi_{m}\right\rangle+\chi_{B\left(\mathbf{R}^{n}\right)}^{m} \circ \ell(s, x, u) \Psi_{m}\right]
$$

gives a Borel function $g(x, t)$ we can define $u_{t}\left(t, \widetilde{\mathbf{X}}_{t}\right)=g\left(t, \widetilde{\mathbf{X}}_{t}\right)$ where $\widetilde{\mathbf{X}}_{t}$ is the unique strong solution of the equation

$$
d \widetilde{\mathbf{X}}_{t}=\chi_{B\left(\mathbf{R}^{n}\right)}^{m} \circ f\left(t, \widetilde{\mathbf{X}}_{t}, g\left(t, \widetilde{\mathbf{X}}_{t}\right)\right) d t+\sigma d \mathbf{B}_{t}
$$

(see the discussion about the SDE at the beginning of this Appendix) and this completes the proof.

At this point we are able to state our last group of conditions. We say that the problem satisfies condition $(\mathbf{H})$ when

$\mathbf{H}$ i. There exists the limit $\Psi$ of $\Psi_{m}$.

$\mathbf{H}$ ii. If

$$
g_{m}(x, t)=\arg \min _{u \in U}\left[\left\langle\chi_{B\left(\mathbf{R}^{n}\right)}^{m} \circ f(s, x, u), \nabla \Psi_{m}\right\rangle+\chi_{B\left(\mathbf{R}^{n}\right)}^{m} \circ \ell(s, x, u) \Psi_{m}\right]
$$

and

$$
g(x, t)=\arg \min _{u \in U}[\langle f(s, x, u), \nabla \Psi\rangle+\ell(s, x, u) \Psi]
$$

then

$$
\lim _{m \rightarrow \infty} \sup _{\|x\|_{\mathbf{R}^{n} \leq K}}\left|\chi_{B\left(\mathbf{R}^{n}\right)}^{m} \circ f\left(t, x, g_{m}(t, x)\right)-f(t, x, g(t, x))\right|=0
$$

for any $K>0$ and all $t \in[0, T]$.

The stated condition will be very useful. It is important to notice that the family of functions $\left\{\chi_{B\left(\mathbf{R}^{n}\right)}^{m}\right\}_{m=1}^{\infty}$ we use has the identity function as limit.

At this point we can prove the following

Lemma 4 There is a constant $B_{1}^{m}$ such that $\left|\log \mathcal{J}^{m}(s, x, u)\right| \leq B_{1}^{m}\left(1+x^{2}\right)$ and there is a constant $B_{1}$, independent from $m$, such that $|\ln \mathcal{J}(s, x, u)| \leq$ $B_{1}\left(1+x^{2}\right)$ for all $s$ and for all $x$.

Proof To start we observe that, by using Gronwall's Inequality ([28]), if we define $Q_{t}=R+K(t-s)+K \int_{s}^{t} Q_{\tau} d \tau+\sigma\left|\mathbf{B}_{t}\right|$ then we have $\left|\mathbf{X}_{t}^{m}\right| \leq Q_{t}$. In fact we know that

$$
d \mathbf{X}_{t}^{m}=\chi_{B\left(\mathbf{R}^{n}\right)}^{m} \circ f\left(t, \mathbf{X}_{t}^{m}, u\right) d t+\sigma d \mathbf{B}_{t}
$$

so, by definition, we have

$$
\mathbf{X}_{t}^{m}=x+\int_{s}^{t} \chi_{B\left(\mathbf{R}^{n}\right)}^{m} \circ f\left(\tau, \mathbf{X}_{\tau}^{m}, u\right) d \tau+\int_{s}^{t} \sigma d \mathbf{B}_{\tau}
$$


and this implies that $\left|\mathbf{X}_{t}^{m}\right| \leq|x|+\int_{s}^{t}\left|\chi_{B\left(\mathbf{R}^{n}\right)}^{m} \circ f\left(\tau, \mathbf{X}_{\tau}^{m}, u\right)\right| d \tau+\sigma\left|\mathbf{B}_{t}\right| \quad$ and if we observe that

$$
\left|\chi_{B\left(\mathbf{R}^{n}\right)}^{m} \circ f\left(\tau, \mathbf{X}_{\tau}^{m}, u\right)\right| \leq K\left(1+\left|\mathbf{X}^{m}\right|\right)
$$

so we have

$$
\begin{aligned}
\left|\mathbf{X}_{t}^{m}\right| & \leq|x|+\int_{s}^{t} K\left(1+\left|\mathbf{X}_{\tau}^{m}\right|\right) d \tau+\sigma\left|\mathbf{B}_{t}\right| \\
& =|x|+\int_{s}^{t} K d \tau+\int_{s}^{t} K\left|\mathbf{X}_{\tau}^{m}\right|+\sigma\left|\mathbf{B}_{t}\right|
\end{aligned}
$$

then the statement follows from Gronwall's Inequality. In the same way we can show that this fact implies that

$$
\left|\mathbf{X}_{t}^{m}-x\right| \leq K(1+|x|) t+K \int_{s}^{t}\left|\mathbf{X}_{\tau}^{m}-x\right| d \tau+\sigma\left|\mathbf{B}_{t}\right|
$$

and we can claim, by Gronwall's Inequality and the Triangle Inequality, that there exists a constant $\widehat{C}$ such that

$$
\begin{aligned}
\left|\mathbf{X}_{t}^{m}\right| & \leq|x|+\widehat{C}\left(K(1+|x|) T+\sigma \max _{0 \leq t \leq T}\left|\mathbf{B}_{t}\right|\right) \\
& \leq(1+|x|)(1+\widehat{C} T K)+\widehat{C} \sigma \max _{0 \leq t \leq T}\left|\mathbf{B}_{t}\right|
\end{aligned}
$$

In the same way, since $\mathbf{X}_{t}$ satisfies

$$
d \mathbf{X}_{t}=f\left(t, \mathbf{X}_{t}, u\right) d t+\sigma d \mathbf{B}_{t}
$$

we have

$$
\mathbf{X}_{t}=x+\int_{s}^{t} f\left(\tau, \mathbf{X}_{\tau}, u\right) d \tau+\int_{s}^{t} \sigma d \mathbf{B}_{\tau}
$$

and this implies that $\left|\mathbf{X}_{t}\right| \leq|x|+\int_{s}^{t}\left|f\left(\tau, \mathbf{X}_{\tau}, u\right)\right| d \tau+\sigma\left|\mathbf{B}_{t}\right|$. If we observe that

$$
\left|f\left(t, \mathbf{X}_{t}, u\right)\right| \leq K\left(1+\left|\mathbf{X}_{t}\right|\right)
$$

then

$$
\begin{aligned}
\left|\mathbf{X}_{t}\right| & \leq|x|+\int_{s}^{t} K\left(1+\left|\mathbf{X}_{\tau}\right|\right) d \tau+\sigma\left|\mathbf{B}_{t}\right| \\
& =|x|+\int_{s}^{t} K d \tau+\int_{s}^{t} K\left|\mathbf{X}_{\tau}\right| d \tau+\sigma\left|\mathbf{B}_{t}\right|
\end{aligned}
$$

hence the statement follows from Gronwall's inequality. In the same way we can show that this fact implies that

$$
\left|\mathbf{X}_{t}-x\right| \leq K(1+|x|) t+K \int_{s}^{t}\left|\mathbf{X}_{\tau}-x\right| d \tau+\sigma\left|\mathbf{B}_{t}\right|
$$


and we have, by Gronwall's Inequality and Triangle Inequality, that there exists a constant $\widehat{C}$ such that

$$
\begin{aligned}
\left|\mathbf{X}_{t}\right| & \leq|x|+\widehat{C}\left(K(1+|x|) T+\sigma \max _{0 \leq t \leq T}\left|\mathbf{B}_{t}\right|\right) \\
& \leq(1+|x|)(1+\widehat{C} T K)+\widehat{C} \sigma \max _{0 \leq t \leq T}\left|\mathbf{B}_{t}\right| .
\end{aligned}
$$

Therefore, if we choose $C=\max \left(K_{1}, K_{2}\right)$ and we assume, without loss of generality, that $s=0$ we get

$$
\begin{aligned}
\left|\int_{0}^{T} \ell\left(\tau, \mathbf{X}_{\iota}, u_{\tau}\right) d \tau+G_{1}\left(\mathbf{X}_{T}\right)\right| \leq & \int_{0}^{T}\left|\ell\left(\tau, \mathbf{X}_{\iota}, u_{\tau}\right)\right| d \tau+\left|G_{1}\left(\mathbf{X}_{T}\right)\right| \\
\leq & \int_{0}^{T} C\left(1+\left|\mathbf{X}_{\tau}\right|^{2}\right) d \tau+C\left(1+\left|\mathbf{X}_{T}\right|^{2}\right) \\
\leq & C(1+T)+C\left|\mathbf{X}_{T}\right|^{2}+C \int_{0}^{T}\left|\mathbf{X}_{\tau}\right|^{2} d \tau \\
\leq & C(1+T)+C\left|\mathbf{X}_{T}\right|^{2} \\
& +C \int_{0}^{T}\left(2\left(1+|x|^{2}(1+\widehat{C} T K)^{2}\right) d \tau\right. \\
& +C \int_{0}^{T}\left(2 \widehat{C}^{2} \sigma^{2}\left(\max _{0 \leq t \leq T}\left|\mathbf{B}_{t}\right|\right)^{2}\right) d \tau \\
\leq & C(1+T)+C\left|\mathbf{X}_{T}\right|^{2} \\
& +C\left(2(1+|x|)^{2}(1+\widehat{C} T K)^{2}\right) T \\
& +2 \widehat{C}^{2} C \sigma^{2} \int_{0}^{T}\left(\max _{0 \leq t \leq T}\left|\mathbf{B}_{t}\right|^{2}\right) d \tau
\end{aligned}
$$

so, after we set

$$
p(|x|, T)=C(1+T)+C\left|\mathbf{X}_{T}\right|^{2}+C\left(2(1+|x|)^{2}(1+\widehat{C} T K)^{2}\right) T
$$

to simplify the notation, we can write

$\mathbf{E}_{0, x} \exp \left\{\left|\varphi_{x, u}(t, \omega)\right|\right\} \leq \exp p(|x|, T) \mathbf{E}_{0, x} \exp \left\{\left|\widehat{C}^{2} C \sigma^{2} \int_{0}^{T}\left(\max _{0 \leq t \leq T}\left|\mathbf{B}_{t}\right|\right)^{2} d t\right|\right\}$

We need to calculate $\mathbf{E}_{0, x} \exp \left\{2 \widehat{C}^{2} C \sigma^{2} \int_{0}^{T}\left(\max _{0 \leq \tau \leq T}\left|\mathbf{B}_{\tau}\right|\right)^{2} d t\right\}$. By using Doob's maximal inequality and by using the fact that the density of $B_{t}^{(j)}$ is 
given by $(2 \pi t)^{-1 / 2} \exp \left(-x^{2} / 2 t\right)$ we get that, for $\sigma$ small enough, the integral is always bounded. In fact, we have the following

$$
\begin{aligned}
\mathbf{E}_{0, x} \exp \left\{\left|\varphi_{x, u}(t, \omega)\right|\right\} & \leq \exp p(|x|, T) \mathbf{E}_{0, x} \exp \left\{\left|\widehat{C}^{2} C \sigma^{2} \int_{0}^{T}\left(\max _{0 \leq t \leq T}\left|\mathbf{B}_{t}\right|\right)^{2} d t\right|\right\} \\
& \leq \frac{2 n}{\sqrt{2 \pi T}} \exp p(|x|, T) \int_{-\infty}^{\infty} \exp \left\{2 \widehat{C}^{2} C \sigma^{2}-\frac{1}{2 T}\right\} w^{2} d w .
\end{aligned}
$$

To complete the proof we notice that by taking the $\log$ of both sides and using the definition of $\mathcal{J}$ then there must exist a constant $B_{1}$ such that $|\ln \mathcal{J}(s, x, u)| \leq B_{1}\left(1+x^{2}\right)$, similarly for $\mathcal{J}^{m}$.

It is useful, in order to prove our next result, to simplify the notation. We set

$$
\varphi_{\mathbf{X}^{m}, x, u}(s, \omega)=\int_{s}^{T} \ell\left(t, \mathbf{X}_{t}^{m}, u_{t}\right) d t+G_{1}\left(\mathbf{X}_{T}^{m}\right)
$$

if

$$
\left\{\begin{array}{l}
d \mathbf{X}_{t}^{m}=\left(\chi_{B\left(\mathbf{R}^{n}\right)}^{m} \circ f\right)\left(t, \mathbf{X}_{t}^{m}, u\right) d t+\sigma d \mathbf{B}_{t} \\
\mathbf{X}_{s}^{m}=x
\end{array} \quad \forall t \in[s, T]\right.
$$

and we set

$$
\left\{\varphi_{\mathbf{X}, x, u}(s, \omega)=\int_{s}^{T} \ell\left(t, \mathbf{X}_{t}, u_{t}\right) d t+G_{1}\left(\mathbf{X}_{T}\right)\right.
$$

if

$$
\left\{\begin{array}{l}
d \mathbf{X}_{t} \\
\mathbf{X}_{s}=x
\end{array} \quad=f\left(t, \mathbf{X}_{t}, u\right) d t+\sigma d \mathbf{B}_{t} \quad \forall t \in[s, T] .\right.
$$

We are now ready to state and prove the following

Lemma 5 We have

$$
\lim _{m \rightarrow \infty}\left(\sup _{u, x}\left|\mathbf{E}_{s, x} \exp \left\{\varphi_{\mathbf{X}^{m}, x, u}(s, \omega)\right\}-\mathbf{E}_{s, x} \exp \{\varphi \mathbf{X}, x, u(s, \omega)\}\right|\right)=0
$$

i.e. we have uniform convergence on compact sets.

Proof Let us fix a compact set $\mathcal{K} \subset \mathbf{R}^{n}$, if we define

$$
B_{f, m}=\sup \left\{c \geq 0 \mid f=\chi_{B\left(\mathbf{R}^{n}\right)}^{m} \circ f \quad \forall(t, x, u) \in[0, T] \times c B\left(\mathbf{R}^{n}\right) \times U\right\}
$$

and

$$
B_{\ell, m}=\sup \left\{c \geq 0 \mid \ell=\chi_{B\left(\mathbf{R}^{n}\right)}^{m} \circ \ell \quad \forall(t, x, u) \in[0, T] \times c B\left(\mathbf{R}^{n}\right) \times U\right\}
$$

and

$$
B_{G_{1}, m}=\sup \left\{c \geq 0 \mid G_{1}=\chi_{B\left(\mathbf{R}^{n}\right)}^{m} \circ G_{1} \forall(t, x, u) \in[0, T] \times c B\left(\mathbf{R}^{n}\right) \times U\right\}
$$


if we consider the following function

$$
D_{m, X, u}^{s, x}=\mathbf{E}_{s, x} \exp \left\{\varphi_{\mathbf{X}_{t}^{m}, u}(t, \omega)\right\}-\mathbf{E}_{s, x} \exp \left\{\varphi_{\mathbf{X}, u}(t, \omega)\right\} .
$$

and if we define

$$
\Omega_{m}=\left\{\omega \in \Omega|| \mathbf{X}_{t}(\omega) \mid \leq \min \left(B_{f, m}, B_{\ell, m}, B_{G_{1}, m}\right) \forall t \in[0, T]\right\}
$$

then, since $X_{t}(\omega)=\mathbf{X}_{t}^{m}(\omega)$ on $\Omega_{m}$, the following inequality

$$
\left|D_{m, X, u}^{s, x}\right| \leq\left|\mathbf{E}_{s, x} \mathbf{1}_{\Omega_{m}^{c}} \exp \left\{\varphi_{\mathbf{X}_{t}^{m}, u}(t, \omega)\right\}+\mathbf{E}_{s, x} \mathbf{1}_{\Omega_{m}^{c}} \exp \left\{\varphi_{\mathbf{X}, u}(t, \omega)\right\}\right|
$$

must hold. Therefore, by the definition of the functions $\varphi_{\mathbf{X}^{m}, x, u}(s, \omega)$ and $\varphi_{\mathbf{X}, x, u}(s, \omega)$ and the properties of the functions $\ell$ and $G_{1}$, we have

$$
\begin{aligned}
\left|D_{m, X, u}^{s, x}\right| \leq & \mathbf{E}_{s, x} \mathbf{1}_{\Omega_{m}^{c}} \exp \left\{\int_{0}^{T} K_{1}\left(1+\left|\mathbf{X}_{t}^{m}\right|^{2}\right) d t+K_{2}\left(1+\left|\mathbf{X}_{T}^{m}\right|^{2}\right)\right\} \\
& +\mathbf{E}_{s, x} \mathbf{1}_{\Omega_{m}^{c}} \exp \left\{\int_{0}^{T} K_{1}\left(1+\left|\mathbf{X}_{t}\right|^{2}\right) d t+K_{2}\left(1+\left|\mathbf{X}_{T}\right|^{2}\right)\right\} .
\end{aligned}
$$

Since we proved in the first part of Lemma 2 that $\left|\mathbf{X}_{t}^{m}\right|$ and $\left|\mathbf{X}_{t}\right|$ are dominated by the process $Q(t)=Q^{x}(t)^{5}$ which is the solution of the equation $Q^{x}(t)=\|x\|+K(t-s)+K \int_{s}^{t} Q^{x}(\tau) d \tau+\sigma\left|\mathbf{B}_{t}\right|$ (note that $\mathcal{K}$ compact implies $\max _{x \in \mathcal{K}}\|x\|$ is a finite positive number) then if we set

$$
c_{1}=(1+T) \max \left(K_{1}, K_{2}\right)
$$

we can write that

$$
\left|D_{m,, X, u}^{s, x}\right| \leq 2 \exp c_{1} \int_{\Omega} \mathbf{1}_{\Omega_{m}^{c}}(\omega) \exp \left\{\frac{c_{1}}{1+T} \int_{0}^{T} Q^{x}(t)^{2}+Q^{x}(t)^{2}\right\} d \mathbf{P}(\omega)
$$

Now we observe that

$$
\mathbf{P}\left(\Omega_{m}^{c}\right) \leq \mathbf{P}\left(\max _{0 \leq t \leq T}\left|Q_{t}\right|>\min \left(B_{f, m}, B_{\ell, m}, B_{G_{1}, m}\right)\right)
$$

and $\min \left(B_{f, m}, B_{\ell, m}, B_{G_{1}, m}\right) \rightarrow \infty$ as $m \rightarrow \infty$. To complete the proof we need to show that $\mathbf{P}\left(\Omega_{m}^{c}\right) \rightarrow 0$ therefore we note that $\left|Q^{x}(t)\right| \leq\|x\|+K T+$ $K \int_{0}^{t}\left|Q^{x}(\tau)\right| d \tau+\sigma \max _{0 \leq t \leq T}\left|\mathbf{B}_{t}\right|$ and the Gronwall's Inequality implies

$$
\begin{aligned}
\left|Q^{x}(t)\right| \leq & \|x\|+K T+\sigma \max _{0 \leq t \leq T}\left|\mathbf{B}_{t}\right|+ \\
& K \int_{s}^{t} \exp K(t-s)\left(\|x\|+K T+\sigma \max _{0 \leq t \leq T}\left|\mathbf{B}_{t}\right|\right) \\
\leq & (1+K T \exp K T)\left(\|x\|+K T+\sigma \max _{0 \leq t \leq T}\left|\mathbf{B}_{t}\right|\right) .
\end{aligned}
$$

\footnotetext{
5 The reader should notice that for the process $Q(t)$ we shall use also the symbol $Q^{x}(t)$. We do this because we need to stress the uniform convergence.
} 
The last estimate implies that

$$
\mathbf{P}\left(\max _{0 \leq t \leq T}\left|Q_{t}\right|>\min \left(B_{f, m}, B_{\ell, m}, B_{G_{1}, m}\right)\right)
$$

is smaller than

$$
\mathbf{P}\left(\|x\|+K T+\sigma \max _{0 \leq t \leq T}\left|\mathbf{B}_{t}\right|>\frac{\min \left(B_{f, m}, B_{\ell, m}, B_{G_{1}, m}\right)}{1+K T \exp K T}\right)
$$

therefore we can conclude that

$$
\mathbf{P}\left(\Omega_{m}^{c}\right)<\mathbf{P}\left(\max _{0 \leq t \leq T}\left|\mathbf{B}_{t}\right|>\frac{\min \left(B_{f, m}, B_{\ell, m}, B_{G_{1}, m}\right)}{\sigma(1+K T \exp K T)}-\frac{1}{\sigma}(\|x\|+K T)\right)
$$

and, since

$$
\lim _{m \rightarrow \infty} \frac{\min \left(B_{f, m}, B_{\ell, m}, B_{G_{1}, m}\right)}{\sigma(1+K T \exp K T)}=\infty
$$

and

$$
\max _{x \in \mathcal{K}}\|x\|<\infty
$$

with $\mathcal{K} \subset \mathbf{R}^{n}$ compact, we can claim that this implies $\mathbf{P}\left(\Omega_{m}^{c}\right) \rightarrow 0$ uniformly.

We also observe that the following inequality

$$
\begin{aligned}
\int_{0}^{T} Q^{x}(t)^{2}+Q^{x}(T)^{2} \leq & \int_{0}^{T}(1+K T \exp K T)^{2}\left(\|x\|+K T+\sigma \max 0 \leq t \leq T\left|\mathbf{B}_{t}\right|\right)^{2} d \tau+ \\
& (1+K T \exp K T)^{2}\left(\|x\|+K T+\sigma \max _{0 \leq t \leq T}\left|\mathbf{B}_{t}\right|\right)^{2} \\
\leq & (1+T)(1+K T \exp K T)^{2} \\
& \times\left(\|x\|+K T+\sigma \max _{0 \leq t \leq T}\left|\mathbf{B}_{t}\right|\right)^{2} \\
\leq & 4(1+T)(1+K T \exp K T)^{2} \\
& \times\left(\|x\|^{2}+K^{2} T^{2}+\sigma^{2}\left(\max _{0 \leq t \leq T}\left|\mathbf{B}_{t}\right|\right)^{2}\right),
\end{aligned}
$$

and the Dominated Convergence Theorem and the Doob's Maximal Inequality imply that

$\lim _{m \rightarrow \infty} 2 \exp c_{1} \int_{\Omega} \mathbf{1}_{\Omega_{m}^{c}}(\omega) \exp \left\{4 c_{1}(1+K T \exp K T)^{2}\left(\|x\|^{2}+K^{2} T^{2}+\sigma\left(\max _{0 \leq t \leq T}\left|\mathbf{B}_{t}\right|\right)^{2}\right)\right\} d \mathbf{P}(\omega)=0$

therefore we can conclude that, uniformly for $x \in \mathcal{K}$,

$$
\lim _{m \rightarrow \infty} 2 \exp c_{1} \int_{\Omega} \mathbf{1}_{\Omega_{m}^{c}}(\omega) \exp \left\{\frac{c_{1}}{1+T} \int_{0}^{T} Q^{x}(t)^{2}+Q^{x}(T)^{2}\right\} d \mathbf{P}(\omega)=0
$$

and this completes the proof. 
Theorem 10 On compact sets we have $V_{m} \rightarrow V$ and $\Psi_{m} \rightarrow \Psi$ uniformly and the value function $V$ satisfies the following problem

$$
0=\frac{\partial V}{\partial t}+\frac{1}{2} \sigma^{2} \Delta V+\frac{1}{2} \sigma^{2}|\nabla V|^{2}+\min _{u \in \mathcal{U}}[\langle f(t, x, v), \nabla V\rangle+\ell(t, x, v)]
$$

Proof If we denote $\log \mathcal{J}_{m}$ by $\mathcal{I}_{m}$ and we remind the reader that we have just proved that

$$
\lim _{m \rightarrow \infty}\left(\sup _{u, x}\left|\mathbf{E}_{s, x} \exp \left\{\varphi_{X^{m}, x, u}(s, \omega)\right\}-\mathbf{E}_{s, x} \exp \left\{\varphi_{X, x, u}(s, \omega)\right\}\right|\right)=0
$$

then we can claim that there is uniform convergence on compact sets for the following sequences

$$
\mathcal{J}_{m} \rightarrow \mathcal{J}
$$

and

$$
\mathcal{I}_{m} \rightarrow \mathcal{I}
$$

To prove that we also have uniform convergence on compact sets for the following sequences

$$
V_{m} \rightarrow V
$$

and

$$
\Psi_{m} \rightarrow \Psi
$$

it is enough to observe that if we fix a $\epsilon>0$ and we fix a compact set $\mathcal{K}$ then, if $m$ is big enough, we have $\left|\mathcal{I}_{m}-\mathcal{I}\right|<\epsilon$ therefore we can find a control $u$ such that

$$
V_{m}(s, x)-V(s, x)<\mathcal{I}_{m}(s, x, u)-\mathcal{I}(s, x, u)+\epsilon
$$

as a consequence we have, for any $x \in K$, the following

$$
V_{m}(s, x)-V(s, x)<2 \epsilon
$$

and this implies that $V_{m} \rightarrow V$ uniformly on compact sets. In the same way we can prove that $\Psi_{m} \rightarrow \Psi$ uniformly on compact sets.

Since we have

$$
\begin{aligned}
0= & \frac{\partial V_{m}}{\partial t}+\frac{1}{2} \sigma^{2} \Delta V_{m}+\frac{1}{2} \sigma^{2}\left|\nabla V_{m}\right|^{2}+ \\
& \min _{u \in \mathcal{U}}\left[\left\langle\chi_{B\left(\mathbf{R}^{n}\right)}^{m} \circ f(t, x, v), \nabla V_{m}\right\rangle+\chi_{B\left(\mathbf{R}^{n}\right)}^{m} \circ \ell(t, x, v)\right]
\end{aligned}
$$

and

$$
0=\frac{\partial V}{\partial t}+\frac{1}{2} \sigma^{2} \Delta V+\frac{1}{2} \sigma^{2}|\nabla V|^{2}+\min _{u \in \mathcal{U}}[\langle f(t, x, v), \nabla V\rangle+\ell(t, x, v)]
$$

if we set

$$
D_{m}=\left[\langle f(t, x, v), \nabla V\rangle+\chi_{B\left(\mathbf{R}^{n}\right)}^{m} \circ \ell(t, x, v)\right]
$$


and

$$
D=[\langle f(t, x, v), \nabla V\rangle+\ell(t, x, v)]
$$

then we can claim $D_{m} \rightarrow D$ uniformly on any compact set $\mathcal{K}$. Therefore, by Lemma 2.6.2 in [8] we can claim that $V$ is the viscosity solution and since we can apply the local existence theorems to the equation with viscosity data we can conclude that the solution of the equation is actually a classical solution. Clearly, the result goes for the function $\Psi$ since this is just a differentiable transform of the function $V$.

We are now in the position to prove the main result. In fact, we have the following

Theorem 11 Given the model $(\mathcal{P} \mathcal{A})$ described at the beginning, if there exists an open set $\mathcal{O}$ such that $\mathbf{X}_{t} \in \mathcal{O}$ and for all $y \in \mathcal{O} \sigma$ is not singular with the inverse matrix satisfying the following condition

$$
\exists \mathcal{G} \in \mathcal{C}^{2}\left(\mathcal{O}, \mathbf{R}^{n}\right) \quad \mathcal{G}_{y}(y)=\sigma^{-1} \quad \forall y \in \mathcal{O}
$$

and $F \circ \mathcal{G}^{-1}, \widetilde{\mathbf{c}}_{f, c, \sigma}\left(t, \mathcal{G}^{-1}, u_{t}\right)$ and the drift term of $\mathcal{G}\left(\mathbf{X}_{t}\right)$ satisfy the assumptions $(\mathbf{F}),(\mathbf{C}),(\mathbf{G})$ and $(\mathbf{H})$ then the solution of the m-truncated problem $(\mathcal{P} \mathcal{A})_{m}$ converges to the solution of $(\mathcal{P} \mathcal{A})$.

Proof If we start with

$$
d \mathbf{X}_{t}=\psi\left(\mathbf{X}_{t}, u_{t}\right) d t+\sigma\left(\mathbf{X}_{t}\right) d \mathbf{B}_{t}
$$

then, by assumption, we can find $\mathcal{G} \in \mathcal{C}^{2}\left(\mathcal{O}, \mathbf{R}^{n}\right)$ such that $D \mathcal{G}(y)=\sigma^{-1}$ $\forall y \in \mathcal{O}$. Hence, if we apply the Ito Formula to

$$
\mathbf{Y}_{t}=\tilde{\sigma} \mathcal{G}\left(\mathbf{X}_{t}\right)
$$

( $\widetilde{\sigma}$ is an arbitrary positive constant) we obtain

$$
\begin{aligned}
d Y_{k, t}= & \widetilde{\sigma} \frac{\partial \mathcal{G}_{k}}{\partial t}\left(t, \mathbf{X}_{t}\right)+\sum_{j} \widetilde{\sigma} \frac{\partial \mathcal{G}_{k}}{\partial x_{j}}\left(t, \mathbf{X}_{t}\right) d X_{j, t}+\frac{1}{2} \sum_{j, i} \widetilde{\sigma} \frac{\partial \mathcal{G}_{k}}{\partial x_{j} \partial x_{i}}\left(t, \mathbf{X}_{t}\right) d X_{i, t} d X_{j, t} \\
& +\sum_{j} \widetilde{\sigma} \frac{\partial \mathcal{G}_{k}}{\partial x_{j}}\left(t, \mathbf{X}_{t}\right) d X_{j, t}+\frac{1}{2} \sum_{j, i} \widetilde{\sigma}^{2} \frac{\partial \mathcal{G}_{k}}{\partial x_{j} \partial x_{i}}\left(t, \mathbf{X}_{t}\right) d X_{i, t} d X_{j, t}
\end{aligned}
$$

where $\mathbf{Y}_{t}=\left(Y_{1, t}, \ldots, Y_{n, t}\right)$ and $\mathbf{X}_{t}=\left(X_{1, t}, \ldots, X_{n, t}\right)$. Therefore, if we set $\widetilde{\sigma} \mathcal{G}=\widetilde{\mathcal{G}}$, and we observe that for any $t \in[0, T]$ we have $\mathbf{X}_{t}: \Omega \rightarrow \mathcal{O}$ and $\widetilde{\mathcal{G}}^{-1}: \mathcal{O} \rightarrow \mathbf{R}^{n}, \widetilde{\mathbf{c}}:[0, T] \times \mathbf{R}^{n} \times U \rightarrow \mathbf{R}$ and $F_{1}: \mathbf{R}^{n} \rightarrow \mathbf{R}$ then, by using $\widetilde{\mathcal{G}}^{-1}$, we can re-write the problem $(\mathcal{P} \mathcal{A})$ and we obtain

$$
\left\{\begin{array}{ccc}
\max _{u \in \mathcal{U}} & \mathbf{E}\left[-\exp -R\left\{F_{1} \circ \widetilde{\mathcal{G}}^{-1}\left(\mathbf{Y}_{T}\right)+\int_{0}^{T} \widetilde{\mathbf{c}}\left(t, \widetilde{\mathcal{G}}^{-1}\left(\mathbf{Y}_{t}\right), u_{t}\right) d t\right\}\right] \\
d \mathbf{Y}_{t}= & \widetilde{\psi}\left(\mathbf{Y}_{t}, u_{t}\right) d t+\widetilde{\sigma} d \mathbf{B}_{t} \\
\mathbf{Y}_{0}= & \widetilde{\mathcal{G}}(x)
\end{array}\right.
$$


and since this problem is equivalent to solving

$$
\left\{\begin{array}{ccc}
\min _{u \in \mathcal{U}} & \log \mathbf{E}\left[\exp -R\left\{F_{1} \circ \widetilde{\mathcal{G}}^{-1}\left(\mathbf{Y}_{T}\right)+\int_{0}^{T} \widetilde{\mathbf{c}}\left(t, \widetilde{\mathcal{G}}^{-1}\left(\mathbf{Y}_{t}\right), u_{t}\right) d t\right\}\right] \\
d \mathbf{Y}_{t}= & \widetilde{\psi}\left(\mathbf{Y}_{t}, u_{t}\right) d t+\widetilde{\sigma} d \mathbf{B}_{t} \\
\mathbf{Y}_{0}= & \widetilde{\mathcal{G}}(x)
\end{array}\right.
$$

then the result follows from what we proved in this section (with $s=0$ ) with

$$
\begin{aligned}
\ell\left(t, \mathbf{Y}_{t}, u_{t}\right)= & -R \widetilde{\mathbf{c}}\left(t, \widetilde{\mathcal{G}}^{-1}\left(\mathbf{Y}_{t}\right), u_{t}\right) \\
= & R c\left(t, \widetilde{\mathcal{G}}^{-1} \mathbf{Y}_{t}, u_{t}\right)-R a\left(t, \widetilde{\mathcal{G}}^{-1} \mathbf{Y}_{t}\right)+ \\
& \frac{R r}{2}\left\|\nabla_{u} c\left(t, \widetilde{\mathcal{G}}^{-1} \mathbf{Y}_{t}, u_{t}\right) D_{u}^{-1} f\left(t, \widetilde{\mathcal{G}}^{-1} \mathbf{Y}_{t}, u_{t}\right) \sigma\left(t, \widetilde{\mathcal{G}}^{-1} \mathbf{Y}_{t}\right)\right\|^{2} \\
G_{1}\left(\mathbf{Y}_{T}\right)= & -R F_{1} \circ \widetilde{\mathcal{G}}^{-1}\left(\mathbf{Y}_{T}\right) \\
= & -R F_{1}\left(\widetilde{\mathcal{G}}^{-1}\left(\mathbf{Y}_{T}\right)\right) .
\end{aligned}
$$

In fact, in Lemma 22 we proved that there exists a sequence of set $\left\{A_{m}\right\}$ such that $\mathbf{P}\left(A_{m}\right) \rightarrow 1$ and for every $(\omega, t) \in A_{m} \times[0, T] \mathbf{Y}^{m}(\omega, t)=\mathbf{Y}(\omega, t)$. Actually, it is enough to set $A_{m}=\Omega_{m}$ where $\Omega_{m}$ is defined exactly as in the cited Lemma i.e.

$\Omega_{m}=\left\{\omega \in \Omega|| Y_{t}(\omega) \mid \leq \min \left(B_{\widetilde{\psi}, m}, B_{\widetilde{\mathbf{c}}\left(\cdot, \widetilde{\mathcal{G}}^{-1}(\cdot), \cdot\right), m}, B_{F_{1} \circ \widetilde{\mathcal{G}}^{-1}, m}\right) \forall t \in[0, T]\right\}^{6}$.

Moreover, we observe that since in the last Theorem we proved that $\mathbf{H} 1$ holds and since we know that if $g_{m}(x, t)$ is the Borel function (see [7]) so defined

$\left.g_{m}(y, s)=\arg \min _{u \in U}\left[\left\langle\chi_{B\left(\mathbf{R}^{n}\right)}^{m} \circ \widetilde{\psi}(s, y, u), \nabla \Psi_{m}\right\rangle+\chi_{B\left(\mathbf{R}^{n}\right)}^{m} \circ\right) \widetilde{\mathbf{c}}\left(s, \widetilde{\mathcal{G}}^{-1}(y), u\right) \Psi_{m}\right]$

then we have $u_{t}\left(t, \tilde{Y}_{t}\right)=g\left(t, \tilde{Y}_{t}\right)$ where $\tilde{Y}_{t}$ is the unique strong solution of the equation $d \widetilde{Y}_{t}=\chi_{B\left(\mathbf{R}^{n}\right)}^{m} \circ \widetilde{\psi}\left(t, \widetilde{Y}_{t}, g\left(t, \widetilde{Y}_{t}\right)\right) d t+\widetilde{\sigma} d \mathbf{B}_{t}$. If we define

$$
\left\langle\chi_{B\left(\mathbf{R}^{n}\right)}^{m} \circ \widetilde{\psi}(s, y, u), \nabla \Psi_{m}\right\rangle+\chi_{B\left(\mathbf{R}^{n}\right)}^{m} \circ \widetilde{\mathbf{c}}\left(s, \widetilde{\mathcal{G}}^{-1}(y), u\right) \Psi_{m}=F_{m}(s, y, u)
$$

and

$$
\left\langle\widetilde{\psi}(s, y, u), \nabla \Psi_{m}\right\rangle+\widetilde{\mathbf{c}}\left(s, \widetilde{\mathcal{G}}^{-1}(y), u\right) \Psi_{m}=F(s, y, u)
$$

then, for any $K>0$ and $\forall t \in[0, T]$, we have

$$
\lim _{m \rightarrow \infty} \sup _{\|y\|_{\mathbf{R}^{n} \leq K}}\left|F_{m}(s, y, u)-F(s, y, u)\right|=0
$$

and this fact implies the convergence of controls.

${ }^{6}$ Of course, by definition we have $\widetilde{\mathbf{c}}\left(\cdot, \widetilde{\mathcal{G}}^{-1}(\cdot), \cdot\right)\left(t, \mathbf{Y}_{t}, u_{t}\right)=\widetilde{\mathbf{c}}\left(t, \widetilde{\mathcal{G}}^{-1}\left(\mathbf{Y}_{t}\right), u_{t}\right)$ 


\section{Appendix II}

Proof (Theorem 5) We are going to present the proof for the diagonal case in detail; the general case is the same, but notationally very messy. Therefore, by assumption, if we define

$$
\widehat{\sigma} \widehat{\sigma}^{\mathrm{T}}=\left[a_{i j}\right]_{i, j=1, \ldots, n}
$$

we are going to assume $a_{i j}=0$ if $i \neq j$. Since we are only going to work on a discrete space or lattice we shall use the following standard approximations for the derivatives

$$
\left\{\begin{array}{cccc}
\varphi_{t} & \mapsto & \frac{1}{\delta}[\varphi(x, t+\delta)-\varphi(x, t)] \\
\varphi_{x_{j}} \text { if } b_{j} \geq 0 \mapsto & \frac{1}{h}\left[\varphi\left(x+h \mathbf{e}_{j}, t+\delta\right)-\varphi(x, t+\delta)\right] \\
\varphi_{x_{i}} & \text { if } b_{j}<0 \mapsto & \frac{1}{h}\left[\varphi(x, t+\delta)-\varphi\left(x-h \mathbf{e}_{j}, t+\delta\right)\right] \\
\varphi_{x_{i} x_{i}} & \mapsto & \frac{1}{h^{2}}\left[\varphi\left(x+h \mathbf{e}_{i}, t+\delta\right)+\varphi\left(x-h \mathbf{e}_{i}, t+\delta\right)-2 \varphi(x)\right]
\end{array}\right.
$$

where $\mathbf{e}_{j}=(0, . ., 1, \ldots 0) \in \mathbf{R}^{n}$ for $j=1, \ldots, n$ (we do not need to compute $\varphi_{x_{i} x_{j}}$ if $i \neq j$ since $a_{i j}=0$ if $i \neq j$ ). Therefore, if we define

$$
W(x, t)=\mathbf{E}_{x}\left[-\exp -R\left\{\widehat{F}\left(\mathbf{X}_{T}\right)+\int_{t}^{T} \widehat{\mathbf{c}}\left(t, \mathbf{X}_{t}, u_{t}\right) d t\right\}\right]
$$

we have

$$
\frac{\partial W}{\partial t}+\sum_{j=1}^{n} \widehat{\psi}(x, u) \frac{\partial W}{\partial x_{j}}(x, t, u)+\frac{1}{2} \sum_{j=1}^{n} a_{j}(x, u) \frac{\partial^{2} W}{\partial x_{j}^{2}}(x, t, u)-\widehat{\mathbf{c}} W(x, t, u)=0
$$

and, after we discretize the partial derivatives, if we define $\widehat{\psi}^{+}=\max (\widehat{\psi}, 0)$ and $\widehat{\psi}^{-}=\max (-\widehat{\psi}, 0)$, we obtain the following

$$
\begin{aligned}
0= & \frac{W^{h, \delta}(x, t+\delta, u)-W^{h, \delta}(x, t, u)}{\delta} \\
& +\sum_{j=1}^{n} \widehat{\psi}^{+}(x, u) \frac{\left(W^{h, \delta}\left(x+h \mathbf{e}_{j}, t+\delta, u\right)-W^{h, \delta}(x, t+\delta, u)\right)}{h} \\
& -\sum_{j=1}^{n} \widehat{\psi}^{-}(x, u) \frac{\left(W^{h, \delta}(x, t+\delta, u)-W^{h, \delta}\left(x-h \mathbf{e}_{j}, t+\delta, u\right)\right)}{h} \\
& +\frac{1}{2} \sum_{j=1}^{n} a_{j j}(x, u) \frac{W^{h, \delta}\left(x+h \mathbf{e}_{j}, t+\delta, u\right)+W^{h, \delta}\left(x-h \mathbf{e}_{j}, t+\delta, u\right)-2 W^{h, \delta}(x, t+\delta, u)}{2 h^{2}} \\
& -R \widehat{\mathbf{c}}(x, n \delta, u) W^{h, \delta}(x, n \delta, u(x, n \delta))
\end{aligned}
$$


with the boundary condition on $W^{h, \delta}$. Hence, we have

$$
\begin{aligned}
& W^{h, \delta}(x, n \delta, u) \\
= & \sum_{j=1}^{n}\left[\frac{\delta}{2 h^{2}} a_{j j}(x)+\widehat{\psi}^{+}\left((x, u(x, n \delta)) \frac{\delta}{h}\right] W^{h, \delta}(x+h, n \delta+\delta, u)\right. \\
& +\sum_{j=1}^{n}\left[\frac{\delta}{2 h^{2}} a_{j j}(x)+\widehat{\psi}^{-}(x, u(x, n \delta)) \frac{\delta}{h}\right] W^{h, \delta}(x-h, n \delta+\delta, u) \\
& +W^{h, \delta}(x, n \delta+\delta, u)\left[1-\sum_{j=1}^{n} \frac{\delta}{h^{2}} a_{j j}(x)-\sum_{j=1}^{n}|\psi(x, u(x, n \delta))| \frac{\delta}{h}\right] \\
& -R c(x, n \delta, u) W^{h, \delta}(x, n \delta, u(x, n \delta)) \delta
\end{aligned}
$$

it follows that, if we define the quantities

$$
\begin{aligned}
\widehat{p}^{h, \delta}(x, x+h, \mid u(x, n \delta)) & =\frac{\delta}{2 h^{2}} a_{j j}(x)+\widehat{\psi}^{+}\left((x, u(x, n \delta)) \frac{\delta}{h}\right. \\
\widehat{p}^{h, \delta}(x, x-h, \mid u(x, n \delta)) & =\frac{\delta}{2 h^{2}} a_{j j}(x)+\widehat{\psi}^{-}(x, u(x, n \delta)) \frac{\delta}{h} \\
\widehat{p}^{h, \delta}(x, x, \mid u(x, n \delta)) & =1-\frac{\delta}{h^{2}} a_{j j}(x)-|\widehat{\psi}(x, u(x, n \delta))| \frac{\delta}{h}
\end{aligned}
$$

we can write

$$
\begin{aligned}
& W^{h, \delta}(x, n \delta, u)(1+R \widehat{\mathbf{c}}(x, n \delta, u) \delta) \\
= & \sum_{y} \widehat{p}^{h, \delta}(x, y, \mid u(x, n \delta)) W^{h, \delta}(x, n \delta+\delta, u)+ \\
& +\widehat{p}^{h, \delta}(x ; x \mid u(x, n \delta)) W^{h, \delta}(x, n \delta+\delta, u)
\end{aligned}
$$

and most importantly, given our boundedness assumptions, we have, for each element in the grid, positive numbers with

$$
\sum_{y} \widehat{p}^{h, \delta}(x, n \delta ; y, n \delta \mid \gamma)+\widehat{p}^{h, \delta}(x, n \delta ; x, n \delta+\delta \mid \gamma)=1 .
$$

Therefore, we can write the discretized optimal control as

$$
\max _{u \in \mathcal{U}} \mathbf{E}_{x, t}^{u}\left[-\exp -R\left\{-\sum \widehat{\mathbf{c}}\left(x_{x, n}^{u, h, \delta}, u\left(x_{x, n}^{u, h, \delta}\right)\right) \Delta t^{h}\left(x_{x, n}^{u, h, \delta}, u\left(x_{x, n}^{u, h, \delta}\right)\right)+\widehat{F}\left(x_{x, n}^{u, h, \delta}\right)\right\}\right]
$$

and we obtain a solution since we are optimizing a continuous function on a compact space. Let $u: G_{h, \delta} \rightarrow \mathbf{R}$ be the solution. So, if we consider the 
probabilities above as transition probabilities, we can write the Markov Chain $\left\{\mathbf{X}^{h, \delta}\right\}$ on the the grid $G_{h, \delta}$. For $\left\{\mathbf{X}^{h, \delta}\right\}$ we have

$$
\left\{\begin{array}{c}
\mathbf{E}_{x, n}^{u, h, \delta} \Delta \mathbf{X}^{h, \delta}=\widehat{\psi} \delta+o(\delta) \\
\operatorname{cov}_{x, n}^{u, h, \delta} \Delta \mathbf{X}^{h, \delta}=\widehat{\sigma} \widehat{\sigma}^{\mathrm{T}}(x) \delta+o(\delta)
\end{array}\right.
$$

and this gives the claimed local consistency.

Remark 7 It is worth noticing that the equation we wrote above could actually allow us to compute the value $W^{h, \delta}(x, n \delta, u)$ by using induction. In fact, on the right side, we have only values of the form $W^{h, \delta}(x, n \delta+\delta, u)$ and this is due to the fact that the spatial derivatives are computed at time $n \delta+\delta$ in the approximation scheme.

Remark 8 It is worth noticing that in the scalar case we have the following standard approximations for the derivatives

$$
\left\{\begin{array}{ccc}
\varphi_{t} & \mapsto & \varphi(x, t+\delta)-\varphi(x, t) / \delta \\
\varphi_{x} \text { if } b \geq 0 \mapsto & \varphi(x+h, t+\delta)-\varphi(x, t+\delta) / h \\
\varphi_{x} \text { it } b<0 \mapsto & \varphi(x, t+\delta)-\varphi(x-h, t+\delta) / h \\
\varphi_{x x} & \mapsto & \{\varphi(x+h, t+\delta)+\varphi(x-h, t+\delta)-2 \varphi(x, t+\delta)\} / h^{2}
\end{array}\right.
$$

and $n=1$ gives us

$$
\frac{\partial W}{\partial t}+b(x, u) \frac{\partial W}{\partial x}(x, t)+\frac{1}{2} \sigma^{2}(x) \frac{\partial^{2} W}{\partial x^{2}}(x, t)-\widetilde{\mathbf{c}} W(x, t)=0
$$

and, after we discretize the partial derivatives, we obtain the following

$$
\begin{aligned}
& W^{h, \delta}(x, n \delta, u) \\
= & {\left[\frac{\delta}{2 h^{2}} \sigma^{2}(x)+b^{+}(x, u(x, n \delta)) \frac{\delta}{h}\right] W^{h, \delta}(x+h, n \delta+\delta, u) } \\
& +\left[\frac{\delta}{2 h^{2}} \sigma^{2}(x)+b^{-}(x, u(x, n \delta)) \frac{\delta}{h}\right] W^{h, \delta}(x-h, n \delta+\delta, u) \\
& +W^{h, \delta}(x, n \delta+\delta, u)\left[1-\frac{\delta}{h^{2}} \sigma^{2}(x)+|b(x, u(x, n \delta))| \frac{\delta}{h}\right] \\
& -R \widetilde{\mathbf{c}}(x, n \delta, u) W^{h, \delta}(x, n \delta, u) \delta
\end{aligned}
$$

So we can write

$$
\begin{aligned}
& W^{h, \delta}(x, n \delta, u)(1+\widetilde{\mathbf{c}}(x, n \delta, u) \delta) \\
= & \sum_{y} \widehat{p}^{h, \delta}(x, n \delta ; y, n \delta \mid u(x, n \delta)) W^{h, \delta}(x, n \delta+\delta, u)+ \\
& +\widehat{p}^{h, \delta}(x, n \delta ; x, n \delta+\delta \mid u(x, n \delta)) W^{h, \delta}(x, n \delta+\delta, u)
\end{aligned}
$$


Remark 9 About the general case $\sigma \sigma^{\mathrm{T}}=\left[a_{i j}\right]_{i, j=1, \ldots, n}$ we are going to use the following discretizations

$$
\left\{\begin{array}{ccc}
\varphi_{t} \quad & \mapsto(x, t+\delta)-\varphi(x, t) / \delta \\
\varphi_{x_{j}} \text { if } \quad b_{j} \geq 0 \mapsto \varphi\left(x+h \mathbf{e}_{j}, t+\delta\right)-\varphi(x, t+\delta) / h \\
\varphi_{x_{i}} \text { if } \quad b_{j}<0 \mapsto \varphi(x, t+\delta)-\varphi\left(x-h \mathbf{e}_{j}, t+\delta\right) / h
\end{array}\right.
$$

and

$$
\left\{\begin{array}{c}
\varphi_{x_{i} x_{j}} \text { if } a_{i j} \geq 0 \mapsto\left(\begin{array}{c}
2 \varphi(x)+\varphi\left(x+h \sum_{k=i, j} \mathbf{e}_{k}, t+\delta\right) \\
-\varphi\left(x-h \sum_{i, j} \mathbf{e}_{k}, t+\delta\right) \\
-\left[\varphi\left(x+h \mathbf{e}_{i}, t+\delta\right)+\varphi\left(x-h \mathbf{e}_{i}, t+\delta\right)\right] / 2 h^{2} \\
-\left[\varphi\left(x+h \mathbf{e}_{j}, t+\delta\right)+\varphi\left(x-h \mathbf{e}_{j}, t+\delta\right) / 2 h^{2}\right.
\end{array}\right) \cdot \frac{1}{2 h^{2}} \\
\varphi_{x_{i} x_{j}} \text { if } a_{i j}<0 \mapsto\left(\begin{array}{c}
-\left[\begin{array}{c}
\left.2 \varphi(x)+\varphi\left(x+h \mathbf{e}_{i}-h \mathbf{e}_{j}, t+\delta\right)\right] \\
-\varphi\left(x-h \mathbf{e}_{i}+h \mathbf{e}_{j}, t+\delta\right)
\end{array}\right] \\
+\left[\varphi\left(x+h \mathbf{e}_{i}, t+\delta\right)+\varphi\left(x-h \mathbf{e}_{i}, t+\delta\right)\right] \\
+\left[\varphi\left(x+h \mathbf{e}_{j}, t+\delta\right)+\varphi\left(x-h \mathbf{e}_{j}, t+\delta\right)\right.
\end{array}\right) \cdot \frac{1}{2 h^{2}}
\end{array}\right.
$$

We now present the following

Proof (Theorem 14) To simplify the notation we define

$$
F_{1}\left(\mathbf{X}_{T}\right)+\int_{0}^{T} \widetilde{c}\left(t, \mathbf{X}_{t}, u_{t}\right) d t=H(\mathbf{X}, u)
$$

and

$$
\chi_{B\left(\mathbf{R}^{n}\right)}^{n} \circ F_{1}\left(\mathbf{X}_{T}\right)+\int_{0}^{T} \chi_{B\left(\mathbf{R}^{n}\right)}^{n} \circ \widetilde{c}\left(t, \mathbf{X}_{t}, u_{t}\right) d t=H_{n}(\mathbf{X}, u)
$$

we can write

$$
\begin{aligned}
\left|\mathbf{E} \exp -R H(\mathbf{X}, u)-\mathbf{E} \exp -R H\left(\mathbf{X}^{n}, u^{n}\right)\right| \leq & \left|\mathbf{E} \exp -R H(\mathbf{X}, u)-\mathbf{E} \exp -R H_{n}\left(\mathbf{X}^{n}, u^{n}\right)\right| \\
& +\left|\mathbf{E} \exp -R H_{n}\left(\mathbf{X}^{n}, u^{n}\right)-\mathbf{E} \exp -R H\left(\mathbf{X}^{n}, u^{n}\right)\right|
\end{aligned}
$$

Given our assumptions A1, A2 and A3 we need only to study the difference $\chi_{B\left(\mathbf{R}^{n}\right)}^{n} \circ c\left(\mathbf{X}_{t}^{n}, u_{t}^{n}\right)-c\left(\mathbf{X}_{t}, u_{t}\right)$. If we fix an $\epsilon>0$ and we observe that for every $n$ we have the solution $\left(\mathbf{X}_{n, t}, u_{n, t}\right)$ of the $n$-truncated problem then we can choose an $n$ big enough such that there is a set $A_{n}$ with $\mathbf{P}\left(A_{n}^{c}\right) \leq \epsilon$ and for every $(\omega, t) \in A_{n} \times[0, T] \mathbf{X}_{n, t}(\omega, t)=\mathbf{X}(\omega, t)$. We also observe that

$$
\begin{aligned}
\left|\chi_{B\left(\mathbf{R}^{n}\right)}^{n} \circ c\left(t, \mathbf{X}_{t}^{n}, u_{t}^{n}\right)-c\left(t, \mathbf{X}_{t}, u_{t}\right)\right|= & \left|\chi_{B\left(\mathbf{R}^{n}\right)}^{n} \circ c\left(t, \mathbf{X}_{t}, u_{t}^{n}\right)-c\left(t, \mathbf{X}_{t}, u_{t}\right)\right| \mathbf{1}_{A_{n}} \\
& +\left|\chi_{B\left(\mathbf{R}^{n}\right)}^{n} \circ c\left(t, \mathbf{X}_{t}^{n}, u_{t}^{n}\right)-c\left(t, \mathbf{X}_{t}, u_{t}\right)\right| \mathbf{1}_{A_{n}^{c}}
\end{aligned}
$$


and

$$
\begin{aligned}
\left|\chi_{B\left(\mathbf{R}^{n}\right)}^{n} \circ c\left(t, \mathbf{X}_{t}^{n}, u_{t}^{n}\right)-c\left(t, \mathbf{X}_{t}, u_{t}\right)\right| \mathbf{1}_{A_{n}^{c}} \leq & \left|\chi_{B\left(\mathbf{R}^{n}\right)}^{n} \circ c\left(t, \mathbf{X}_{t}^{n}, u_{t}^{n}\right)\right| \mathbf{1}_{A_{n}^{c}} \\
& +\left|c\left(t, \mathbf{X}_{t}, u_{t}\right)\right| \mathbf{1}_{A_{n}^{c}} \\
\leq & 2 K\left(1+\left|\mathbf{X}_{t}^{n}\right|^{2}+\left|\mathbf{X}_{t}\right|^{2}\right) \mathbf{1}_{A_{n}^{c}} \\
\leq & 2 K\left(1+2\left|\mathbf{X}_{t}^{n}-\mathbf{X}_{t}\right|^{2}+3\left|\mathbf{X}_{t}\right|^{2}\right) \mathbf{1}_{A_{n}^{c}}
\end{aligned}
$$

if we define

$$
Y_{n}=\sup _{[0, T]}\left|\mathbf{X}_{t}^{n}-\mathbf{X}_{t}\right|^{2}
$$

we know that (see [10])

$$
Y_{n} \rightarrow 0
$$

so we can conclude $Y_{n} \rightarrow 0$ in probability and $\mathbf{1}_{A_{n}^{c}} \int_{0}^{T}\left|\mathbf{X}_{t}^{n}-\mathbf{X}_{t}\right|^{2} \leq B$ a.e. on $\Omega$, we also notice that there exists a constant $L>0$ such that, if we set

$$
\left|\mathbf{E} \exp \int_{0}^{T} \chi_{B\left(\mathbf{R}^{n}\right)}^{n} \circ c\left(t, \mathbf{X}_{t}^{n}, u_{t}^{n}\right) d t-\mathbf{E} \exp \int_{0}^{T} c\left(t, \mathbf{X}_{t}, u_{t}\right) d t\right|=\mathcal{I},
$$

we have, by A3,

$$
\begin{aligned}
\mathcal{I} & \leq L \mathbf{E}\left|\exp \int_{0}^{T}\right| \chi_{B\left(\mathbf{R}^{n}\right)}^{n} \circ c\left(t, \mathbf{X}_{t}^{n}, u_{t}^{n}\right)-c\left(t, \mathbf{X}_{t}^{n}, u_{t}^{n}\right)\left|\mathbf{1}_{A_{n}^{c}} d t-1\right|^{2} \\
& \leq L \mathbf{E}\left|\exp \int_{0}^{T} 2 K\left(1+2\left|\mathbf{X}_{t}^{n}-\mathbf{X}_{t}\right|^{2}+3\left|\mathbf{X}_{t}\right|^{2}\right) \mathbf{1}_{A_{n}^{c}} d t-1\right|^{2} \\
& \leq\left. L \mathbf{E}\left|\exp \mathbf{1}_{A_{n}^{c}}\left(T+4 K \int_{0}^{T}\left|\mathbf{X}_{t}^{n}-\mathbf{X}_{t}\right|^{2} d t\right)+6 K \mathbf{1}_{A_{n}^{c}} \int_{0}^{T}\right| \mathbf{X}_{t}\right|^{2} d t-\left.1\right|^{2}
\end{aligned}
$$

We also notice that for every $\alpha \in \mathbf{R}$ we have, by Fubini 's Theorem,

$$
\begin{aligned}
\mathbf{E} \exp \alpha \mathbf{1}_{A_{n}^{c}} \int_{0}^{T}\left|\mathbf{X}_{t}^{n}-\mathbf{X}_{t}\right|^{2} d t & =\mathbf{E} \sum_{\ell=0}^{\infty} \frac{1}{\ell !} \alpha^{\ell}\left(\mathbf{1}_{A_{n}^{c}} \int_{0}^{T}\left|\mathbf{X}_{t}^{n}-\mathbf{X}_{t}\right|^{2} d t\right)^{\ell} \\
& =\sum_{\ell=0}^{\infty} \frac{1}{\ell !} \alpha^{\ell} \mathbf{E}\left(\mathbf{1}_{A_{n}^{c}} \int_{0}^{T}\left|\mathbf{X}_{t}^{n}-\mathbf{X}_{t}\right|^{2} d t\right)^{\ell} \\
& \leq \sum_{\ell=0}^{\infty} \frac{1}{\ell !} \alpha^{\ell} B^{\ell} \\
& <\infty
\end{aligned}
$$

and this implies, by the Dominated Convergence Theorem, that

$$
\left|\mathbf{E} \exp \int_{0}^{T} \chi_{B\left(\mathbf{R}^{n}\right)}^{n} \circ c\left(t, \mathbf{X}_{t}^{n}, u_{t}^{n}\right) d t-\mathbf{E} \exp \int_{0}^{T} c\left(t, \mathbf{X}_{t}, u_{t}\right) d t\right| \rightarrow 0
$$


since $\mathbf{P}\left(A_{n}^{c}\right) \rightarrow 0$ and $\mathbf{P}(\Omega)=1$. Finally, we notice that for $\left(\mathbf{X}_{n}^{h_{n}, \delta_{n}}, u_{n}^{h_{n}, \delta_{n}}\right)$ and $\left(\mathbf{X}_{t}^{n}, u_{t}^{n}\right)$ we can use the weak convergence and therefore we can claim that

$\left|\mathbf{E}\left[\exp -R\left\{F\left(\mathbf{X}_{n}^{h_{n}, \delta_{n}}\right)+\int_{0}^{T} c\left(t, \mathbf{X}_{n}^{h_{n}, \delta_{n}}, u_{n}^{h_{n}, \delta_{n}}\right) d t\right\}\right]-\mathbf{E} \exp H\left(\mathbf{X}^{n}, u^{n}\right)\right| \rightarrow 0$

and this completes the proof.

Proof (Theorem 16) We fix an $\epsilon>0$ and we observe that for every $m$ we have the solution $\left(\mathbf{X}_{t}^{m}, u_{t}^{m}\right)$ of the $m$-truncated problem. If we choose an $m$ big enough then there exists an $A_{m}$ such that $\mathbf{P}\left(A_{m}^{c}\right) \leq \epsilon$ and for every $(\omega, t) \in A_{m} \times[0, T] \mathbf{X}_{t}^{m}(\omega, t)=\mathbf{X}_{t}(\omega, t)$. To prove our result we must analyze the different parts of the sharing rule.

We start by proving that $\int_{0}^{T} c\left(t, \mathbf{X}_{t}^{m}, u_{t}^{m}\right) d t \rightarrow \int_{0}^{T} c\left(t, \mathbf{X}_{t}, u_{t}^{*}\right) d t$. We observe that we can write

$$
\begin{aligned}
\left|c\left(t, \mathbf{X}_{t}, u_{t}^{*}\right)-c\left(t, \mathbf{X}_{t}^{m}, u_{t}^{m}\right)\right| \leq & \left.\mid c\left(t, \mathbf{X}_{t}, u_{t}^{*}\right)-c\left(t, \mathbf{X}_{t}^{m}, u_{t}^{*}\right)\right) \mid+ \\
& \left.\mid c\left(t, \mathbf{X}_{t}^{m}, u_{t}^{*}\right)\right)-c\left(t, \mathbf{X}_{t}^{m}, u_{t}^{m}\right) \mid
\end{aligned}
$$

and

$$
\left.\left.\mid c\left(t, \mathbf{X}_{t}, u_{t}^{*}\right)-c\left(t, \mathbf{X}_{t}^{m}, u_{t}^{*}\right)\right)\left|\leq\left(1+\left|\mathbf{X}_{t}\right|+\mid \mathbf{X}_{t}^{m}\right)\right|\right)\left|\mathbf{X}_{t}-\mathbf{X}_{t}^{m}\right|
$$

therefore, by Jensen's Inequality, we have

$$
\begin{aligned}
\left.\mathbf{E} \mid \int_{0}^{T}\left(c\left(t, \mathbf{X}_{t}, u_{t}^{*}\right)-c\left(t, \mathbf{X}_{t}^{m}, u_{t}^{*}\right)\right)\right)\left.d t\right|^{2} & \left.\leq \mathbf{E} \int_{0}^{T} \mid c\left(t, \mathbf{X}_{t}, u_{t}^{*}\right)-c\left(t, \mathbf{X}_{t}^{m}, u_{t}^{*}\right)\right)\left.\right|^{2} d t \\
& \leq \mathbf{E} \int_{0}^{T}\left(\left(1+\left|\mathbf{X}_{t}\right|+\mid \mathbf{X}_{t}^{m}\right) \mid\right)^{2}\left|\mathbf{X}_{t}-\mathbf{X}_{t}^{m}\right|^{2} d t \\
& \leq 4 \mathbf{E} \int_{0}^{T}\left(1+\left|\mathbf{X}_{t}\right|^{2}+\left|\mathbf{X}_{t}^{m}\right|^{2}\right)\left|\mathbf{X}_{t}-\mathbf{X}_{t}^{m}\right|^{2} d t
\end{aligned}
$$

and hence, by Cauchy-Schwarz's Inequality,

$$
\begin{aligned}
\left.\mathbf{E} \mid \int_{0}^{T}\left(c\left(t, \mathbf{X}_{t}, u_{t}^{*}\right)-c\left(t, \mathbf{X}_{t}^{m}, u_{t}^{*}\right)\right)\right)\left.d t\right|^{2} \leq & 4\left(\mathbf{E} \int_{0}^{T}\left(1+\left|\mathbf{X}_{t}\right|^{2}+\left|\mathbf{X}_{t}^{m}\right|^{2}\right)^{2} d t\right)^{\frac{1}{2}} \\
& \times\left(\mathbf{E} \int_{0}^{T}\left|\mathbf{X}_{t}-\mathbf{X}_{t}^{m}\right|^{4} d t\right)^{\frac{1}{2}} \\
\leq & 16\left(\mathbf{E} \int_{0}^{T}\left(1+\left|\mathbf{X}_{t}\right|^{4}+\left|\mathbf{X}_{t}^{m}\right|^{4}\right) d t\right)^{\frac{1}{2}} \\
& \times\left(\mathbf{E} \int_{0}^{T}\left|\mathbf{X}_{t}-\mathbf{X}_{t}^{m}\right|^{4} d t\right)^{\frac{1}{2}}
\end{aligned}
$$


and the last term goes to zero as $m \rightarrow \infty$. We also notice that, by condition $i v$ in Section 3, there exists $K_{1}$ such that

$$
\begin{aligned}
\left.\mid c\left(t, \mathbf{X}_{t}^{m}, u_{t}^{*}\right)\right)-c\left(t, \mathbf{X}_{t}^{m}, u_{t}^{m}\right) \mid \leq & \left.\mid c\left(t, \mathbf{X}_{t}^{m}, u_{t}^{*}\right)\right)-\chi_{B\left(\mathbf{R}^{n}\right)}^{m} \circ c\left(t, \mathbf{X}_{t}^{m}, u_{t}^{m}\right) \mid \\
& \left.+\mid \chi_{B\left(\mathbf{R}^{n}\right)}^{m} \circ c\left(t, \mathbf{X}_{t}^{m}, u_{t}^{*}\right)\right)-c\left(t, \mathbf{X}_{t}^{m}, u_{t}^{m}\right) \mid \\
\leq & 2 K_{1}\left(1+\left|\mathbf{X}_{t}^{m}\right|^{2}\right)
\end{aligned}
$$

and since there exists a constant $K_{2}$ such that (see [10] Theorem 3.8)

$$
\begin{aligned}
\mathbf{E} \int_{0}^{T}\left(1+\left|\mathbf{X}_{t}^{m}\right|^{2}\right)^{2} & =\int_{0}^{T} \mathbf{E}\left(1+\left|\mathbf{X}_{t}^{m}\right|^{2}\right)^{2} \\
& \leq 2 \int_{0}^{T} \mathbf{E}\left(1+\left|\mathbf{X}_{t}^{m}\right|^{4}\right) \\
& \leq 2 \int_{0}^{T}\left(1+(1+x) \exp K_{2} t\right) \\
& <\infty
\end{aligned}
$$

therefore, by the Dominated Convergence Theorem, we obtain that

$$
\left.\mathbf{E} \int_{0}^{T} \mid c\left(t, \mathbf{X}_{t}, u_{t}^{*}\right)-c\left(t, \mathbf{X}_{t}^{m}, u_{t}^{*}\right)\right)\left.\right|^{2} d t \rightarrow 0 \text { as } m \rightarrow \infty
$$

and this implies the convergence of both terms. Now if we use the fact that $\left(\nabla_{u} c \cdot D_{u}^{-1} f\right) \cdot \sigma$ is bounded and the fact that, for a finite constant $C_{1}$,

$$
\begin{aligned}
\mathbf{E}\left[\int_{0}^{T}\left|\mathbf{X}_{t}^{m}(\omega, t)-\mathbf{X}_{t}(\omega, t)\right|^{2} d t\right] & \leq \int_{\Omega} \int_{0}^{T} \mathbf{1}_{A_{m}^{c}}\left|\mathbf{X}_{t}^{m}(\omega, t)-\mathbf{X}_{t}(\omega, t)\right|^{2} d t d \mathbf{P} \\
& \leq C_{1} \mathbf{P}\left(A_{m}^{c}\right) T
\end{aligned}
$$

it follows that, in the $L^{2}$ sense,

$$
\int_{0}^{T}\left(\nabla_{u} c \cdot D_{u}^{-1} f\right)\left(\mathbf{X}_{t}^{m}, u_{t}^{m}\right) \sigma\left(\mathbf{X}_{t}^{m}\right) d \mathbf{B}_{t} \rightarrow \int_{0}^{T}\left(\nabla_{u} c \cdot D_{u}^{-1} f\right)\left(\mathbf{X}_{t}, u_{t}^{*}\right) \sigma\left(\mathbf{X}_{t}\right) d \mathbf{B}_{t}
$$

If we set

$$
I=\left\|\left(\nabla_{u} c \cdot D_{u}^{-1} f\right)\left(\mathbf{X}_{t}^{m}, u_{t}^{m}\right) \sigma\left(\mathbf{X}_{t}^{m}\right)\right\|^{2} d t
$$

then there exists a constant $M$ such that

$$
\begin{aligned}
& \mathbf{E}\left|\int_{0}^{T} I-\left[\int_{0}^{T}\left|\nabla_{u} c\left(t, \mathbf{X}_{t}, u_{t}\right) D_{u}^{-1} f\left(t, \mathbf{X}_{t}, u_{t}\right) \sigma\left(t, \mathbf{X}_{t}\right)\right|^{2} d t\right]\right|^{2} \\
\leq & \mathbf{E}\left|\int_{0}^{T}\left(I-\left|\nabla_{u} c\left(t, \mathbf{X}_{t}, u_{t}\right) D_{u}^{-1} f\left(t, \mathbf{X}_{t}, u_{t}\right) \sigma\left(t, \mathbf{X}_{t}\right)\right|^{2}\right) d t\right|^{2} \\
\leq & \mathbf{E}\left|\int_{0}^{T}\left(I-\left|\nabla_{u} c\left(t, \mathbf{X}_{t}, u_{t}\right) D_{u}^{-1} f\left(t, \mathbf{X}_{t}, u_{t}\right) \sigma\left(t, \mathbf{X}_{t}\right)\right|^{2}\right)\right|^{2} d t \\
\leq & 2 M^{2} T
\end{aligned}
$$


and this implies that

$$
\int_{0}^{T}\left\|\left(\nabla_{u} c \cdot D_{u}^{-1} f\right)\left(t, \mathbf{X}_{t}^{m}, u_{t}^{m}\right) \sigma\left(t, \mathbf{X}_{t}^{m}\right)\right\|^{2}
$$

converges, in the $L^{2}$ norm, to

$$
\int_{0}^{T}\left\|\left(\nabla_{u} c \cdot D_{u}^{-1} f\right)\left(t, \mathbf{X}_{t}, u_{t}^{*}\right) \sigma\left(t, \mathbf{X}_{t}\right)\right\|^{2}
$$

Finally, for any $m$ we have $\left\{\left(\mathbf{X}_{m}^{h_{n}, \delta_{n}}, u_{m}^{h_{n}, \delta_{n}}\right)\right\}_{n}$ and, by using Skorokhod Theorem, we can assume that there is convergence with probability one. Therefore, by the boundedness of the functions $\nabla_{u} c D_{u}^{-1} f \sigma$ and $\chi_{B\left(\mathbf{R}^{n}\right)}^{m} \circ c$ we have clearly the following

$$
\int_{0}^{T} \chi_{B\left(\mathbf{R}^{n}\right)}^{m} \circ c\left(t, \mathbf{X}_{m}^{h_{n}, \delta_{n}}, u_{m}^{h_{n}, \delta_{n}}\right) d t \rightarrow \int_{0}^{T} \chi_{B\left(\mathbf{R}^{n}\right)}^{m} \circ c\left(t, \mathbf{X}_{t}^{m}, u_{t}^{m}\right) d t
$$

and

$$
\int_{0}^{T}\left(\nabla_{u} c \cdot D_{u}^{-1} f\right)\left(t, \mathbf{X}_{m}^{h_{n}, \delta_{n}}, u_{m}^{h_{n}, \delta_{n}}\right) \sigma\left(t, \mathbf{X}_{m}^{h_{n}, \delta_{n}}\right) d \mathbf{B}_{t}
$$

converges to

$$
\int_{0}^{T}\left(\nabla_{u} c \cdot D_{u}^{-1} f\right)\left(t, \mathbf{X}_{t}^{m}, u_{t}^{m}\right) \sigma\left(t, \mathbf{X}_{t}^{m}\right) d \mathbf{B}_{t}
$$

and

$$
\int_{0}^{T}\left\|\left(\nabla_{u} c \cdot D_{u}^{-1} f\right)\left(t, \mathbf{X}_{m}^{h_{n}, \delta_{n}}, u_{m}^{h_{n}, \delta_{n}}\right) \sigma\left(t, \mathbf{X}_{m}^{h_{n}, \delta_{n}}\right)\right\|^{2} d t
$$

converges

$$
\int_{0}^{T}\left\|\left(\nabla_{u} c \cdot D_{u}^{-1} f\right)\left(t, \mathbf{X}_{t}^{m}, u_{t}^{m}\right) \sigma\left(t, \mathbf{X}_{t}^{m}\right)\right\|^{2} d t
$$

therefore we obtain

$$
L^{2}-\lim _{n \rightarrow \infty} S\left(\mathbf{X}_{n}\right)=S(\mathbf{X})
$$




\section{References}

1. Anderson, Robert M. and Roberto C. Raimondo, "Market Clearing and Derivative Pricing," Economic Theory 25(2005), 21-34. .

2. Billingsley, P., Convergence of Probability Measures; Second Edition, Wiley, New York, 1999.

3. Biais, B. and M. Mariotti, G. Plantin and J.-C. Rochet, "Dynamic Security Design: Convergence to Continuous Time and Asset Pricing Implications" The Review of Economic Studies, vol. 74, n. 2, April 2007, p. 345-390.

4. Davis, M.H.A., "Martingale Methods in Stochastic Control," in Stochastic Differential Systems (M. Kohlmann and W. Vogel, Eds) pp. 85-111, Lecture Notes in Control and Information Science, Vol 16, Springer Verlag, New York, 1979.

5. Dixit, A. K. and R. W. Pindyck, Investment under Uncertainty, Princeton University Press, Princeton NJ, 1994.

6. Elliott, R.J., Stochastic Calculus and Applications, Springer, New York and Berlin, 1982.

7. Fleming, W. H. and R. W. Rishel, Deterministic and Stochastic Optimal Control, App. of Mathematics Vol. 1 Springer, New York and Berlin, 1975.

8. Fleming, W. H. and H. M. Soner, Controlled Markov Processes and Viscosity Solutions, Springer, New York and Berlin, 1992.

9. Fleming, W. H. and S. J. Shieu, Risk-sensitive Control and Optimal Investment problem II, The Annals of Applied Probability, 12 (2002), No. 2 730-767.

10. Gard, T. C., Stochastic Differential Equations, Dekker, New York 1988.

11. Gihman, I..I. and A.V. Skorokhod, Stochastic Differential Equations, Springer, New York 1972.

12. Hellwig, M. and K. Schmidt, "Discrete-Time Approximations of the HolmströmMilgrom Brownian Motion Model of Intertemporal Incentive Provision," Econometrica 70 (2002), 2225-2264.

13. Holmström , B. and P. Milgrom, "Moral hazard and Observability," Bell Journal of Econ., 10 (1979), 74-91.

14. Holmström , B. and P. Milgrom, "Aggregation and Linearity in the Provision of Intertemporal Incentives," Econometrica, 55 (1987), 303-328.

15. Kaplan, T., "Communication of Preferences in Contests for Contracts," Economic Theory, Forthcoming

16. Karni, E., "Agency Theory: Choice-based Foundations of the Parametrized Distribution Formulation," Economic Theory 36 (2008), 337-351.

17. Krylov, N.V., Controlled Diffusion Processes, Springer, 1980.

18. Kurtz, H.J., Approximation of Population Processes, Vol. 36 of CBMS-NSF Regional Conf. in Appl. Math. SIAM Philadelphia, 1981.

19. Kushner, H.J., Numerical Methods for Stochastic Control Problems in Continuous Time, Springer-Verlag, New York and Berlin, 1982.

20. Kushner, H.J., Numerical Methods for Stochastic Control Problems in Finance. In M.A.H. Dempster and S.R. Pliska, editors, Mathematics of Derivative Securities, pages 504-527. Cambridge University Press, Cambrigde, UK, 1997.

21. Ladyzenskaya,O.A. and V.A. Solonnikov and N.N. Uralceva, Linear and Quasi-linear Equations of Parabolic Type, American Mathematical Society, Rhodes Island (USA), 1968.

22. Laffont, J.-J. and D. Martimort, The Theory of Incentives: The Principal-Agent Model, Princeton University Press, 2002.

23. LiCalzi, M. and S. Spaeter, "Distributions for the First-Order Approach to PrincipalAgent Problems," Economic Theory, 21 (2003), no. 1, 167-173.

24. Mirrlees, J., "An Exploration in the Theory of Optimal Income Taxation" Review of Economic Studies, 38 (1971), 175-208.

25. Mirrlees, J., "Note on Welfare Economics, Information and Uncertainty" in Essays on Behavior Under Uncertainty, ed. by M. Balch, D. McFadden, S. Wu. Amsterdam: North Holland, 243-258 (1974).

26. Mirrlees, J., "The Theory of Moral Hazard and Unobservable Behavior: Part I," Review of Economic Studies, 66 (1999), 3-22. 
27. Mirrlees, J., "The Optimal Structure of Incentives and Authority within an Organization," Bell Journal of Economics, 7 (1976), 105-131.

28. Øksendal, B., Stochastic Differential Equations, $6^{\text {th }}$ Edition, Springer, Berlin, 2010.

29. Protter, P., Stochastic Integration and Differential Equations, Springer, Berlin, 2005.

30. Raimondo, R. C., "Market Clearing, Utility Functions, and Securities Prices," Economic Theory, 25 (2005), 265-285.

31. Rogerson, W. , "The First-Order approach to Principal-Agent Problems," Econometrica 53 (1985), 1357-1367.

32. Sannikov, Y., "A Continuous-Time Version of the Principal-Agent Problem." Review of Economic Studies (2008), 75: 957-984.

33. Schaettler, H. and J. Sung, "The First Order Approach to the Continuous-Time Principal Agent Problem with Exponential Utility" J. of Econ. Theory, 61 (2000), 331-371.

34. Sung, J., "Corporate Insurance and Managerial Incentives" J. of Econ. Theory, 61(1997), 297-332.

35. Sung, J., "Managerial Compensation and Corporate Management Under Moral Hazard" PhD Thesis, Washington University St. Louis, 1991.

36. Sung, J., "Optimal Contracts under Moral Hazard and Adverse Selection" UIC working paper, 2000. 


\section{University Library}

\section{- M M I E E R VA A gateway to Melbourne's research publications}

Minerva Access is the Institutional Repository of The University of Melbourne

Author/s:

Mirrlees, J;Raimondo, RC

Title:

Strategies in the principal-agent model

Date:

2013-08-01

Citation:

Mirrlees, J. \& Raimondo, R. C. (2013). Strategies in the principal-agent model. ECONOMIC THEORY, 53 (3), pp.605-656. https://doi.org/10.1007/s00199-012-0706-2.

Persistent Link:

http://hdl.handle.net/11343/282684 